\title{
Thomas d'Aquin, l' étiologie proclusienne et la théorie du concours de Dieu à la causalité naturelle
}

\author{
Jean-Luc Solère \\ PSL, LEM UMR 8584 / Boston College
}

Selon Thomas d'Aquin, Dieu n'a pas seulement créé au commencement. Il reste à l' œuvre à tout moment subséquent pour donner l' existence à toute nouvelle créature issue de processus naturels, c' est-à-dire de processus impliquant des causes qui sont elles-mêmes des créatures. Sans son action qui sous-tend celle des causes secondes, ces dernières seraient impuissantes à produire quoi que ce soit'1.

Cette thèse sur la causalité divine a été marquée par l' étiologie proclusienne, que Thomas a découverte d'abord dans le Livre des causes, puis redécouverte dans les Éléments de théologie directement ${ }^{2}$ - non pas qu' elle ait été son unique source, mais il est certain que Thomas l'a abondamment citée ou utilisée. Le Livre des causes pose en effet, dès son premier chapitre, le principe général qu' une cause première influe davantage sur ses effets que les causes prochaines de ces mêmes effets ${ }^{3}$. Alors même qu' une cause seconde communique sa pro-

1 De plus, selon la thèse de la conservation ou création continuée, Dieu maintient constamment dans l'être, non seulement le monde dans son ensemble, mais aussi chaque créature individuellement, après qu' elle a été produite et en chaque instant aussi longtemps qu' elle existe. Je laisserai ici de côté cette thèse, pour me consacrer à celle du concours de Dieu aux actions des causes secondes. Il faut noter, cependant, que cette dernière peut être déduite de la première, comme par exemple dans l'argument de Summa contra Gentiles, III, 67.3: Sicut autem Deus non solum dedit esse rebus cum primo esse incoeperunt, sed quandiu sunt, esse in eis causat, res in esse conservans, ut ostensum est, ita non solum cum primo res conditae sunt eis virtutes operativas dedit, sed semper eas in rebus causat. Unde, cessante influentia divina, omnis operatio cessaret. Omnis igitur rei operatio in ipsum reducitur sicut in causam. Mais ce n' est pas le seul argument, et je centrerai cette étude sur ceux qui, plus directement, établissent la thèse du concours à partir du statut particulier de la donation d'être.

2 Comme on sait, c' est tardivement que Thomas a pu prendre connaissance des Éléments de théologie (à partir de 1268, lorsque Guillaume de Moerbeke les a traduits en latin), et a en conséquence découvert la source principale du Livre des causes (voir le prooemium de sa Super Librum de causis expositio, écrite vers 1271-1272, p. 3, l. 3-5). Mais l'opuscule, dont le prestige était grand jusqu' alors, a façonné sa pensée dans ses années de formation et au long de la plus grande partie de sa carrière.

3 Liber de causis, I, 1, p. 38: Omnis causa primaria plus est influens super causatum suum quam

(C) KONINKLIJKE BRILL NV, LEIDEN, THE NETHERLANDS, 2022 | DOI:10.1163/9789004501331_014

This is an open access chapter distributed under the terms of the CC BY-NC 4.o license. 
priété à son effet, elle ne le fait pas indépendamment de la cause première: «lorsque la cause seconde produit une chose, la cause première, qui est audessus d' elle, influe sur cette chose de par sa propre puissance ${ }^{4}$. Une raison décisive pour cela est que donner l'être est la causalité propre de la Cause première: «si toute cause donne à son effet quelque chose, dans ce cas, sans aucun doute, l'être premier donne l'être à tous les effets ${ }^{5}$. Si la cause première n' agissait pas en sous-main, une cause seconde n'aurait simplement aucun effet. En conséquence, la cause première seule est dite agir par création, alors que les causes secondes agissent par (in)formation, c' est à dire par adjonction de propriétés à un sujet qu'elles ne produisent pas ${ }^{6}$.

Ces idées, si importantes soient-elles comme fondements de la théorie du concours divin, qui joua un rôle majeur dans la métaphysique médiévale et encore dans la métaphysique du XVII ${ }^{\mathrm{e}}$ siècle, sont hautement contre-intuitives. Que toute nouvelle existence soit produite par Dieu seulement semble contraire à toute l'évidence empirique. Les composants d'une synthèse chimique paraissent bien en produire le résultat; plantes et animaux paraissent bien causer l'existence de leur descendance. De plus, si la cause première érige les créatures en causes, pourquoi donc ne les érigerait-elle pas en causes efficientes de plein droit? Pourquoi les causes secondes ne pourraient-elles produire elles-mêmes l'existence de leur effet? Pourquoi ce pouvoir causal ne pourrait-il être donné aux causes secondes, parmi tous les autres biens que la cause première leur dispense? Le nier ne revient-il pas tout simplement à diminuer la causalité des créatures? N'est-ce pas tomber, sinon dans l'occasionalisme, du moins dans un régime où l'action des causes secondes n'est que fantomatique? Néanmoins, Thomas d'Aquin soutient bien que puisque tout processus naturel, même un changement accidentel (par exemple une altération), consiste à amener à l'être (par exemple amener à être-chaud, i.e. actualiser la forme accidentelle de chaleur dans le patient $)^{7}$, et puisque rien

causa universalis secunda. Liber de causis, I.18, p. 40: (...) causa longinqua est vehementius causa rei quam causa propinqua (...). Voir les remarques de D'Ancona 2014, p. 143, sur le fait que le Livre commence avec ce qui correspond à la proposition $5^{6}$ des Éléments. Selon elle, c' est un choix délibéré de l'Adaptateur: «instead of the opposition one-multiple, it is the primacy of the remote, transcendent First Cause that sets the tone in the Liber de causis».

4 Liber de causis, I, 17, p. 40: (...) causa secunda quando facit rem, influit causa prima quae est supra eam super illam rem de virtute sua (...).

5 Liber de causis, XVII, 144, p. 66: (...) si omnis causa dat causato suo aliquid, tunc procul dubio ens primum dat causatis omnibus ens.

6 Liber de causis, XVII, 148, p. 66.

7 Thomas d'Aquin, Quaestiones disputate de potentia, q. 7, a. 2, c.: Omnes autem causae creatae 
ne saurait donner l' être sans l' aide de Dieu, aucun processus naturel ne peut être accompli sans le concours divin 8 .

Je me propose de montrer dans ce qui suit comment Thomas d'Aquin explique, justifie, complète, utilise, et au besoin corrige, ces thèses paradoxales. Nous examinerons d'abord les arguments qui montrent que Dieu est cause immédiate de toute existence. Puis nous verrons comment Thomas élucide, au moyen du modèle de la causalité instrumentale, le rapport entre cette action de la cause première et les actions des causes secondes ${ }^{9}$.

communicant in uno effectu qui est esse (...). Calor enim facit calidum esse, et aedificator facit domum esse.

8 Thomas d'Aquin, Summa contra Gentiles, III, 67.1: Omne enim operans est aliquo modo causa essendi, vel secundum esse substantiale, vel accidentale. Nihil autem est causa essendi nisi inquantum agit in virtute Dei. On pourrait penser que puisque les causes naturelles ne produisent pas ex nihilo mais s' exercent toujours sur un matériau pré-donnée, cela est la raison pour laquelle elles ne donnent pas l'existence: l'existence est déjà donnée avec la matière. Mais ce serait négliger le fait que, pour Thomas, c' est la forme qui donne l' existence ( forma dat esse). Ce n' est donc pas le cas que l' existence du substrat pré-donné devienne l' existence de ce qui est engendré. Dans la mesure où l'agent actualise une nouvelle forme (substantielle ou accidentelle), il actualise une nouvelle existence (substantielle ou accidentelle), même s'il y avait dans le substrat la puissance de devenir ce qui est engendré (Thomas d' Aquin, De principiis naturae, cap. 1: Sed materia habet esse ex eo quod ei advenit, quia de se habet esse incompletum. Unde, simpliciter loquendo, forma dat esse materiae (...). Sicut autem omne quod est in potentia potest dici materia, ita omne a quo aliquid habet esse, quodcumque esse sit sive substantiale, sive accidentale, potest diciforma; sicut homo cum sit potentia albus, fit actu albus per albedinem, et sperma, cum sit potentia homo, fit actu homo per animam).

9 Je me limiterai ici à la question de la donation de l'existence aux effets des causes secondes. Un autre aspect de la théorie du concours divin, déjà visible dans la doctrine du Livre des causes, est le fait que Dieu assiste la causalité des causes subordonnées même pour ce qu' elles ont à faire en propre. Cela s' applique entre autres à la volonté humaine: aucune volition n' est possible sans le concours de Dieu; Dieu est cause non seulement de la faculté, mais aussi des actes de cette faculté (voir par exemple Thomas d'Aquin, Summa contra Gentiles, III, 67.4, et III, 89.5: Deus non solum dat rebus virtutes, sed etiam nulla res potest propria virtute agere nisi agat in virtute ipsius, ut supra ostensum est. Ergo homo non potest virtute voluntatis sibi data uti nisi inquantum agit in virtute Dei. Illud autem in cuius virtute agens agit, est causa non solum virtutis, sed etiam actus. Quod in artifice apparet, in cuius virtute agit instrumentum, etiam quod ab hoc artifice propriam formam non accepit, sed solum ab ipso applicatur ad actum. Deus igitur est causa nobis non solum voluntatis, sed etiam volendi.). 
Des arguments par lesquels Thomas démontre que toute donation d' existence, à tout moment, provient de la cause première, le plus connu, et qui s'appuie directement sur le Livre des causes, est celui donné dans la Summa theologiae $^{10}$ :

1. Plus un effet est universel (c' est-à-dire, répandu), plus sa cause est universelle (a une action étendue) et est élevée (c' est-à-dire primitive dans la série des causes).

2. Or, l'effet le plus universel de tous est l' existence (esse).

3. Donc l' existence doit être l' effet propre de la cause la plus universelle et la plus élevée, Dieu.

La mineure, 2, repose sur la constatation tautologique que toutes les choses qui sont produites ont au moins en commun l' existence, le fait d'être, quelles que soient leurs natures. Thomas fait suivre la conclusion qui en découle par une référence synthétique au Livre des causes (principalement proposition III): ni l'Intelligence ni l'Âme noble ne communiquent l' existence, si ce n'est en tant qu' elles sont agies par l'opération divine. En revanche, il ne mentionne pas que la majeure, 1, repose sur le théorème posé par Proclus: «ce qui cause un plus grand nombre d' effets est supérieur à ce qui en cause moins » ${ }^{11}$, qui est une application du principe général que «toute cause opère avant ses effets et donne lieu à un plus grand nombre de conséquences $»^{12}$. Autrement dit, la causalité de la cause la plus haute est celle qui produit le plus d'effets et descend jusqu' au plus bas de la hiérarchie, à savoir, la matière. Les causes inférieures ont une action plus limitée, proportionnellement à leur place dans la hiérarchie. Ce principe n' est pas explicité dans le De causis, mais est mentionné en passant au début («la cause première lointaine embrasse davantage» que les causes subordonnées ${ }^{13}$ ), et est présupposé dans la démonstration de II.23-24: la cause première est «avant» ou «au-dessus » de l'éternité, car puisque l'éternité suppose l'être, l'être est plus commun que l'éternité et la cause de l'être est donc

10 Thomas d'Aquin, Summa theologiae, p. I, q. 45, a. 5, c.: Oportet enim universaliores effectus in universaliores et priores causas reducere. Inter omnes autem effectus, universalissimum est ipsum esse. Unde oportet quod sit proprius effectus primae et universalissimae causae, quae est Deus. Unde etiam dicitur Libro de causis, quod neque intelligentia vel anima nobilis dat esse, nisi inquantum operatur operatione divina.

11 Proclus, Éléments de théologie, 6o, p. 58.

12 Proclus, Éléments de théologie, 57, p. 54. Voir aussi Proclus, Éléments de théologie, 138, p. 122.

13 Liber de causis, I.12, p. 40: (...) causa prima longinqua est plus comprehendens (...). 
supérieure à l'éternitél ${ }^{14}$. Par contre, Thomas fait appel explicitement à ce principe en expliquant la proposition I dans son commentaire du De causis ${ }^{15}$.

Un argument du De potentia remplace l'universalité de l' existence par son caractère de fondement des autres propriétés ${ }^{16}$ :

1. L'ordre des effets est calqué sur l'ordre des causes: à cause subordonnée, effet subordonné (et à effet subordonné, cause subordonnée).

2. Or l'existence est un effet qui n'en présuppose aucun autre, mais, au contraire, est présupposé par tous les autres, car tout effet ne peut être effet que s' il existe.

3. L'existence est donc l' effet tout premier, dans l'ordre des conditions.

4. Par conséquent, l' existence doit être produite par la cause première et seulement par elle.

La mineure 2 peut être rapprochée de la proposition I du De causis, qui explique que la propriété d'être (effet propre de la cause première) est (onto)logiquement présupposée par la propriété de vivre ou de penser (effets de causes secondes), alors que l'inverse n' est pas vrai ${ }^{17}$. Le De causis en donne une illustration concrète: dans la génération d'un être humain, l' existence du substrat matériel est donnée en premier; vient ensuite la vie ou animalité; vient enfin la rationalité qui le rend humain ${ }^{18}$. Inversement, dans l'ordre de la corruption il perd d' abord l' usage de la raison, alors qu' il demeure vivant et respire encore; puis, il ne reste que l' existence, celle du corps inanimé. L' action de la cause première est donc plus fondamentale que celle de la cause seconde: elle la précède et subsiste après elle; elle en est donc indépendante. C' est pourquoi la cause première est davantage liée à un effet que la cause prochaine de celui-ci, et en est plus véritablement cause ${ }^{19}$.

\footnotetext{
14 Liber de causis, II.23-24, p. 42. Cf. Proclus, Éléments de théologie, 87, p. 80.

15 Thomas d' Aquin, Super Librum de causis expositio, p. 8, 1. 21-22.

16 Thomas d'Aquin, De potentia, 3.4.c.: (...) ordo effectuum est secundum ordinem causarum. Primus autem effectus est ipsum esse, quod omnibus aliis effectibus praesupponitur et ipsum non praesupponit aliquem alium effectum; et ideo oportet quod dare esse in quantum huiusmodi sit effectus primae causae solius secundum propriam virtutem (...). Voir aussi Thomas d'Aquin, Summa contra Gentiles, III, 66.6, et De substantiis separatis, c. 10.

17 Liber de causis, I.8 et I.10, p. 38.

18 Liber de causis, I.6-11, p. 38-40. Cf. Proclus, Éléments de théologie, 7o, p. 66, 1. 18-22. Thomas, dans son commentaire, souligne qu'Aristote soutient la même chose dans De generatione animalium, II.3, 736a 24: l' individu est animal avant que d'être humain.

19 Liber de causis, I.12, I.13 et I.18, p. 40. «Plus fortement cause», vehementius causa, dit exactement le texte (cf. Proclus, Éléments de théologie, 56, p. 54, l. 5 et 21: aitioterôn, meizonôs aition), parce qu' elle «s' attache plus fortement à la chose que l' opération de la cause prochaine», ou «adhère» en elle d'une adhérence plus forte.
} 
Thomas fournit bien d'autres démonstrations, qui ne réfèrent pas directement au Livre des causes. Je signalerai simplement les suivantes, qui utilisent un type de raisonnement que l' on pourrait qualifier de "platonicien». Ainsi, un autre argument du De potentia choisit la route suivante ${ }^{20}$ :

1. Ce qui est commun à une pluralité ne peut appartenir à aucun des membres de cette pluralité en raison de sa nature propre, car la nature propre de chaque membre est ce en raison de quoi il se distingue des autres, non ce en raison de quoi il a quelque chose de commun avec les autres.

2. Il faut donc que cette propriété commune vienne d'une cause qui soit extérieure à cette pluralité.

3. Or toutes les créatures (c' est-à-dire des êtres qui n'existent pas par nature) ont en commun de recevoir l'être.

4. Par conséquent, leur existence doit provenir d' une cause qui n' est aucune des créatures.

La Summa contra Gentiles offre un argument que, de même, on peut appeler platonicien parce qu'il repose sur la notion de participation ${ }^{21}$ :

1. Ce qui a une propriété par essence est cause de ce qui a cette propriété par participation; ainsi, l'élément feu est cause de toute incandescence dans les choses.

2. Or Dieu est être (ens) par essence.

3. Il ne peut y avoir qu' un seul être de ce type (par Summa contra Gentiles, I.42).

4. Par conséquent, Dieu est cause de l' existence de toutes les autres choses, qui obtiennent l'être par participation.

Enfin, toujours dans la Summa contra Gentiles, Thomas démontre ainsi qu' il ne peut y avoir aucune chose qui ne tienne son existence de $\mathrm{Dieu}^{22}$ :

20 Thomas d'Aquin, De potentia 3.6.c.: Oportet enim, si aliquid unum communiter in pluribus invenitur, quod ab aliqua una causa in illis causetur; non enim potest esse quod illud commune utrique ex se ipso conveniat, cum utrumque, secundum quod ipsum est, ab altero distinguantur; et diversitas causarum diversos effectus producit. Cum ergo esse inveniatur omnibus rebus commune, quae, secundum illud quod sunt, ad invicem distinctae sunt, oportet quod de necessitate eis non ex se ipsis, sed ab aliqua una causa esse attribuatur.

21 Thomas d'Aquin, Summa contra Gentiles, II.15.5: Quod per essentiam dicitur, est causa omnium quae per participationem dicuntur: sicut ignis est causa omnium ignitorum inquantum huiusmodi. Deus autem est ens per essentiam suam: quia est ipsum esse. Omne autem aliud ens est ens per participationem: quia ens quod sit suum esse non potest esse nisi unum ut in primo ostensum est. Deus igitur est causa essendi omnibus aliis. Voir aussi Summa contra Gentiles, III, 66.7.

22 Thomas d'Aquin, Summa contra Gentiles, II.15.2: Omne enim quod alicui convenit non secundum quod ipsum est, per aliquam causam convenit ei, sicut album homini: nam quod 
1. Une propriété qui est présente dans une chose mais ne lui appartient pas en vertu de sa propre nature, doit avoir été donnée à cette chose par une cause (il doit en effet y avoir une raison de la présence de cette propriété).

2. Il est impossible que deux choses différentes aient une même propriété chacune en raison de leurs natures propres respectives, car ce qui découle d'une nature propre ne s'étend pas au-delà des bornes de cette nature (par exemple, avoir trois angles dont la somme est égale à deux droits n' appartient qu' aux triangles).

3. Si donc deux choses ont une même propriété, l'une des deux au moins doit avoir reçu cette propriété d'une cause autre que sa propre nature.

Deux possibilités s' offrent alors:

3.1. L'une de ces deux choses est cause de la présence de la propriété dans l'autre.

3.2. Toutes les deux ont reçu cette propriété d' une troisième chose.

4. Or, tout ce qui est a la propriété d' exister (esse).

5. Donc (par 3), de deux choses qui existent, l'une au moins doit avoir reçu l' existence d'une cause; c' est-à-dire (par 3.1 et 3.2):

5.1. Ou bien l'une est cause de l' existence de l'autre,

5.2. Ou bien toutes deux existent à cause d'une troisième.

6. Mais Dieu est un être qui n'a reçu l' existence d'aucune cause.

7. Donc (par 5.1), prenant Dieu et n'importe lequel des autres êtres, Dieu doit être cause de l'existence de cet autre être, puisqu'il est impossible que cet être soit la cause de Dieu, ou que Dieu et cet être reçoivent tous deux l' existence à cause d'un autre être.

causam non habet, primum et immediatum est, unde necesse est ut sit per se et secundum quod ipsum. Impossibile est autem aliquod unum duobus convenire et utrique secundum quod ipsum. Quod enim de aliquo secundum quod ipsum dicitur, ipsum non excedit: sicut habere tres angulos duobus rectis aequales non excedit triangulum. Si igitur aliquid duobus conveniat, non conveniet utrique secundum quod ipsum est. Impossibile est igitur aliquod unum de duobus praedicari ita quod de neutro per causam dicatur, sed oportet vel unum esse alterius causam, sicut ignis est causa caloris corpori mixto, cum tamen utrumque calidum dicatur; vel oportet quod aliquod tertium sit causa utrique, sicut duabus candelis ignis est causa lucendi. Esse autem dicitur de omni eo quod est. Impossibile est igitur esse aliqua duo quorum neutrum habeat causam essendi, sed oportet utrumque acceptorum esse per causam, vel alterum alteri esse causam essendi. Oportet igitur quod ab illo cui nihil est causa essendi, sit omne illud quod quocumque modo est. Deum autem supra ostendimus huiusmodi ens esse cui nihil sit causa essendi. Ab eo igitur est omne quod quocumque modo est. Si autem dicatur quod ens non est praedicatum univocum, nihil minus praedicta conclusio sequitur. Non enim de multis aequivoce dicitur, sed per analogiam: et sic oportet fieri reductionem in unum. 
On remarquera que toutes ces démonstrations ont en commun de requérir Dieu non pas comme cause première, point de départ, d'une série causale, mais comme cause présente et immédiate de chaque existant individuellement, rejoignant en cela la doctrine du De causis. Toute existence doit venir directement de la cause première; par conséquent, Dieu produit à tout moment l'existence de tout effet, c' est-à-dire de tout ce qui vient à l'être (substances, accidents, actions, etc.)

\section{La coopération entre la cause première et les causes secondes}

Étant admis que l'être de tous les effets vient directement de Dieu, l'on doit maintenant se demander quel est le rôle des causes secondes dans les processus naturels (par opposition à une venue à l'être surnaturelle, si par exemple Dieu créait, en cet instant, un veau à partir de rien). Un certain nombre de contraintes sont à respecter, ce qui rend la réponse difficile à élaborer philosophiquement ${ }^{23}$.

\subsection{Les réquisits}

(R1) Premièrement, dans un processus naturel il faut que la cause créée ait une action bien à elle, qui soit l'expression de sa nature propre; sinon, ce serait en vain qu' elle aurait cette nature ${ }^{24}$. Si elle n'opérait rien de son propre cru, elle ne serait pas du tout cause ${ }^{25}$. D' une manière générale, Thomas essaie de situer sa théorie de la causalité comme une troisième voie entre deux opposés. Un extrême est de ne pas donner assez aux créatures, en leur refusant toute causalité efficiente et en faisant de Dieu la seule cause efficiente, comme dans l'occasionalisme des Mutakalimun - connu de Thomas via Al-Ghazali et Maïmonide, et qu' il rejette. L'autre extrême est de donner trop aux créatures, en leur conférant une causalité indépendante de Dieu, voire, comme Avicenne, la possibilité de créer à leur tour, par délégation du pouvoir divin. La doctrine du

\footnotetext{
23 Voir Freddoso 1988; Freddoso 1991; Freddoso 1994.

24 Thomas d'Aquin, De potentia 3.7.c.: Non ergo sic est intelligendum quod Deus in omni re naturali operetur, quasi res naturalis nihil operetur; sed quia in ipsa natura vel voluntate operante Deus operatur.

25 Thomas d'Aquin, Summa theologiae, I, 45.5.c.: (...) causa secunda instrumentalis non participat actionem causae superioris, nisi inquantum per aliquid sibi proprium dispositive operatur ad effectum principalis agentis. Si igitur nihil ibi ageret secundum illud quod est sibi proprium, frustra adhiberetur ad agendum, nec oporteret esse determinata instrumenta determinatarum actionum.
} 
Livre des causes aide Thomas à tracer cette via media, dans la mesure où elle admet une spécificité de l' action des créatures, tout en la subordonnant à celle de Dieu.

(R2) Cependant, cet agencement de causalités doit obéir à une seconde contrainte: Dieu et la créature doivent coopérer au même effet au sein d'une seule et même action. Il n'y pas de double engendrement d'un veau, par Dieu et par une vache. Bien plutôt, il n'y a qu'un seul engendrement. L' effet ne résulte donc pas de deux actions, certes liées mais seulement parallèles. Il n'y a pas non plus deux effets. Une unique substance vivante, individuelle, est engendrée, comme un tout. Un seul et même effet est donc le résultat de l'action conjointe de Dieu et de la cause seconde.

(R3) Ensuite, ni l' action de Dieu ni celle de la créature ne doivent être redondantes. Autrement dit, aucune des deux causes n'est suffisante, car chacune est nécessaire (du moins en régime ordinaire, car, par puissance absolue, Dieu pourrait bien sûr agir seul s'il le voulait).

$\left(\mathrm{R}_{4}\right)$ Toutefois, quatrième contrainte, les deux causes doivent être hiérarchiquement ordonnées. Elles ne sont pas deux causes collaborant au même niveau. Non seulement la causalité de Dieu doit être cause de celle de la créature, mais encore elle doit être d'un ordre supérieur, c' est-à-dire doit enrôler celle de la créature, plutôt que l' inverse.

$\left(R_{5}\right)$ Enfin, dernière contrainte: Dieu ne cause pas une partie de l' effet et la créature une autre partie. La causalité de chacune des deux causes doit être intégrale à l' égard de l' effet - ceci pour respecter les droits de la causalité naturelle: lorsqu' une vache engendre un veau, elle n' engendre pas seulement une partie de ce veau. Autrement dit, chacune des deux causes est une cause totale, c' est-à-dire une cause du tout. Une cause totale n' est pas une cause suffisante, car, justement, dans la théorie de Thomas les créatures sont causes totales de leurs effets, mais non causes suffisantes. Cause totale s' oppose à cause partielle. Exemples de causes partielles: un maçon et un charpentier construisant chacun une partie d'une maison, ou deux personnes portant un même fardeau. Exemple de cause totale: plusieurs personnes collaborant à un projet. Leurs compétences peuvent être diverses, mais dans la mesure où elles contribuent toutes au projet dans son ensemble (au lieu que, par exemple, telle personne écrive une partie d'un livre, telle autre une autre partie), elles sont causes totales. De plus elles peuvent être subordonnées les unes aux autres, s'il y a une hiérarchie entre les collaborateurs ${ }^{26}$.

26 Cf. Thomas d' Aquin, Summa contra Gentiles, III, 67.5: (...) in rebus voluntariis omnes artifices inferiores operantur secundum imperium supremi architectoris. 
Pour résumer toutes ces contraintes en une seule formule: il doit y avoir deux causes réelles, qui soient hiérarchiquement ordonnées, qui soient toutes deux causes totales, et qui agissent dans une même action.

\subsection{Théorie de la causalité instrumentale}

Comment est-il possible que deux causalités s' interpénètrent à ce point, sans se gêner l' une l' autre pour ainsi dire, ce qui est précisément la doctrine du De causis? Le modèle auquel Thomas recourt pour l' expliquer est celui de la causalité instrumentale. Ce modèle repose, comme Thomas le souligne, sur la distinction entre, d' une part, la chose qui agit, et d' autre part, le pouvoir en vertu duquel cette chose agit ${ }^{27}$.

Tournons-nous vers le De potentia, q. 3, a. 7, où Thomas explique qu'une chose peut être dite cause de l'action d'une autre en quatre différents sens.

(S1) Premièrement, une chose est cause de l'action d'une autre quand elle donne à cette autre chose son pouvoir d'action (virtus operandi). C'est ainsi que ce qui engendre quelque chose qui est lourd ou léger, lui donne le pouvoir de monter ou de descendre par un mouvement naturel. C'est évidemment le cas aussi pour la cause première à l' égard des causes secondes.

(S2) Deuxièmement, ce qui préserve un pouvoir contre la corruption peut être dit cause de l' action d'une autre chose, comme lorsqu' on dit que des médicaments qui préservent la vue font voir.

$\left(\mathrm{S}_{3}\right)$ Troisièmement, une chose peut être dite cause de l'action d'une autre en tant qu' elle meut cette chose à agir. C' est un sens beaucoup plus fort que la donation ou la conservation d' un pouvoir d' agir: cela consiste en «l'application» de ce pouvoir, comme, dit Thomas, lorsque le pouvoir de couper d'un couteau est actualisé par notre force physique ${ }^{28}$. Notons bien qu'ici la cause principale n' a pas à agir sur la nature même de la cause seconde. Certes, on peut dire que c' est lorsqu' elle est produite et acquiert sa forme qu' une chose devient un instrument, au moins en puissance, comme lorsque del'acier façonné d'une certaine manière devient lame de couteau. Mais une chose devient actuellement un instrument lorsqu' elle est mise en action par la cause principale ${ }^{29}$. Il peut arriver en effet que bien que la cause seconde soit complètement déter-

27 Thomas d'Aquin, Summa contra Gentiles, III, 70.5: In quolibet enim agente est duo considerare, scilicet rem ipsam quae agit, et virtutem qua agit (...).

28 Thomas d'Aquin, De potentia, 3.7.c.: Sed quia nulla res per se ipsam movet vel agit nisi sit movens non motum, tertio modo dicitur una res esse causa actionis alterius in quantum movet eam ad agendum; in quo non intelligitur collatio aut conservatio virtutis activae, sed applicatio virtutis ad actionem; sicut homo est causa incisionis cultelli ex hoc ipso quod applicat acumen cultelli ad incidendum movendo ipsum.

Cf. Thomas d'Aquin, Summa theologiae, III, 72.3, ad 2: Instrumentum virtutem instrumen- 
minée, c' est-à-dire possède déjà ses propres forme et type d'action, la mise en acte de son pouvoir nécessite l'opération d' une cause supérieure. Cette cause supérieure peut n' être pas la cause productrice de cette cause instrumentale (comme lorsque j'utilise un couteau que je n'ai pas fabriqué); mais elle est requise pour actualiser son pouvoir causal, ce qu' elle fait en se subordonnant le pouvoir de cette dernière et en le déterminant à produire un certain effet ${ }^{30}$. Bien sûr, rien n'empêche que la cause principale soit aussi cause efficiente de la cause instrumentale (comme lorsque celui qui fabrique un outil en est aussi l'utilisateur). Mais ce qui importe ici, dans cette étape de l'analyse de Thomas, c' est qu'une cause est instrumentale quand son pouvoir est conjoint à son effet par le pouvoir d'une autre cause. Il est donc de la nature d'une cause instrumentale d'être un moteur mû, comme le dit Thomas ${ }^{31}$. La cause seconde ainsi mise en mouvement est un instrument, au sens large, de la cause qui la met en mouvement. L'exemple du couteau, proposé par Thomas, ne doit toutefois pas nous faire penser que «l'application» de la cause seconde par la cause principale se limite à une mise en mouvement au sens littéral. Il est des forces naturelles qui agissent d'elles-mêmes, mais qui deviennent instrumentales dans la mesure où elles sont utilisées par une cause supérieure. Pour prendre un exemple médiéval, un meunier ne met pas le vent en mouvement, mais le vent devient bien cause instrumentale de la mouture du blé lorsque le mécanisme du moulin est soumis à sa force motrice. L'idée qui se trouve derrière les notions d'application ou de mise en mouvement est que la cause directrice actualise le pouvoir de la cause secondaire en l'attelant, pour ainsi dire, à la production de son effet.

(S4) Ce n'est pas tout. En quatrième lieu, une cause supérieure peut se joindre à l'action d' une cause subordonnée et lui faire faire quelque chose que cette cause subordonnée ne pourrait pas faire par elle-même. D'une manière générale, toute cause seconde peut être le siège d'une double action causale: celle qu' elle exerce en vertu de sa nature propre, et celle qu' elle exerce en vertu d' une cause première ${ }^{32}$. Mais une cause instrumentale en ce sens est un cas particulier de cause seconde dans lequel, sous l'impulsion de la cause principale,

talem acquirit dupliciter, scilicet quando accipit formam instrumenti, et quando movetur a principali agente ad effectum.

30 Cf. Thomas d' Aquin, Summa theologiae, III, 72.3, ad 2: Instrumentum movetur a principali agente ad effectum.

31 Cf. Thomas d'Aquin, Summa contra Gentiles, II, 21.4: Est ratio instrumenti quod sit movens motum. Thomas d'Aquin, Summa theologiae III, 63.5, ad 2: Ratio instrumenti consistit in hoc quod ab alio moveatur, non autem in hoc quod ipsum se moveat.

32 Cf. Thomas d'Aquin, De potentia, 3.4, c.: (...) causa secunda duplicem actionem habere potest: unam ex propria natura, aliam ex virtutis prioris causae. 
par cette nouvelle action la cause subordonnée produit quelque chose qui est au-delà de ce que ce qu' elle peut faire de par sa nature propre, et qui a pour fin l' effet de la cause principale ${ }^{33}$. Par exemple, en physique-chimie aristotélicienne, par leurs propriétés telles que chaleur, froideur, sécheresse, humidité, les éléments feu, air, eau, terre ont certaines actions spécifiques sur la matière: froideur ou sécheresse la contractent, etc. Mais en tant que ces propriétés se trouvent dans une substance qui est composée desdits éléments, elles sont les causes instrumentales de l' action de cette substance sur une autre chose. C'est en effet toujours par l'intermédiaire de ces qualités accidentelles que la forme substantielle d'un agent commence d'agir sur un patient. Cette action aboutit à l' actualisation de la forme substantielle de l' agent dans le patient, résultat que les qualités élémentaires ne pourraient produire d' elles-mêmes ${ }^{34}$. Ainsi un feu, par l' intermédiaire de sa chaleur, finit par actualiser la forme de feu dans une buche. La chaleur a pour effet propre de dessécher, non pas d'enflammer. Seul le feu le peut. Mais il le fait à travers l'action de sa chaleur.

Certes, une distinction entre causes instrumentales s' impose ${ }^{35}$. Dans certains cas, la cause instrumentale participe directement à la production de cet effet de la cause principale. Par exemple, la chaleur a pour effet naturel de dissoudre et consumer; mais en tant que son pouvoir est utilisé par une âme végétative pour la digestion des nutriments, son action aboutit à la fabrica-

33 Dvořák 2013, p. 620-622, insiste à bon escient sur ce point en commentant le même texte. Voir aussi Thomas d'Aquin, De veritate, 27.4, ad 2: (...) sacramenta novae legis non sunt causa gratiae principalis, quasi per se agentia, sed causa instrumentalis. Et secundum modum aliorum instrumentorum habent duplicem actionem: unam quae excedit formam propriam, sed est ex virtute formae principalis agentis, scilicet Dei: quae est iustificare; et aliam quam exercet secundum formam propriam, sicut abluere vel ungere (...).

34 Thomas d'Aquin, Quaestiones disputatae de anima 12, c.: Quia enim agens naturale in generatione agit transmutando materiam ad formam, quod quidem fit secundum quod materia primo disponitur ad formam, et tandem consequitur formam, secundum quod generatio est terminus alterationis; necesse est quod ex parte agentis id quod immediate agit sit forma accidentalis correspondens dispositioni materiae. Sed oportet ut forma accidentalis agat in virtute formae substantialis, quasi instrumentum eius; alias non induceret agendo formam substantialem. Et propter hoc in elementis non apparent aliqua principia actionum, nisi qualitates activae et passivae, quae tamen agunt in virtute formarum substantialium. Et propter hoc earum actio non solum terminatur ad dispositiones accidentales, sed etiam ad formas substantiales. Nam et in artificialibus actio instrumenti terminatur ad formam intentam ab artifice.

35 Thomas d'Aquin, Scriptum super Sententiis, IV, d. 1, q. 1, a. 4, 1, c.: (...) causa efficiens dupliciter potest dividi. Uno modo ex parte effectus; scilicet in disponentem, quae causat dispositionem ad formam ultimam; et perficientem, quae inducit ultimam perfectionem. Alio modo ex parte ipsius causae in agens principale, et instrumentale. 
tion de la chair, qui est l'effet propre de l'âme végétative ${ }^{36}$. Dans d'autres cas, l' action de la cause instrumentale s' arrête à la préparation du patient sur lequel la cause principale agit, et elle ne contribue pas directement à l' effet de celleci (par exemple, la chaleur qui contribue à la fabrication d'un corps humain ne fait que préparer ce dernier à la réception de l'âme, laquelle n' est pas une forme matérielle et n' est causée que par Dieu) ${ }^{37}$. Mais même dans ce cas, tout comme dans le premier, l' action instrumentale de la cause subordonnée, la préparation même du sujet, sous l' action de la cause principale, et en vue de l' action finale faite par la seule cause principale, va au-delà de ce que la cause subordonnée est capable de faire lorsqu' elle n' est pas dirigée par une cause principale ${ }^{38}$; sinon, dit Thomas, «elle n' agirait pas comme un instrument» 39 .

Il faut noter que, dans tous les cas, l'action instrumentale est une action additionnelle, qui se superpose à celle que la cause subordonnée exécute par elle-même en vertu de sa nature et de ses pouvoirs causaux ${ }^{40}$. Deux actions prennent donc place simultanément dans la cause subordonnée: celle qui cor-

36 Voir Aristote, De anima, II.4, 416b28-29. Thomas d' Aquin, Scriptum super Sententiis, IV, d. 1, q. 1, a. 4, 1, c.: Instrumento autem competit duplex actio: una quam habet ex propria natura, alia quam habet prout est motum a primo agente; sicut calor ignis, qui est instrumentum virtutis nutritivae, ut dicitur in 2 de anima, ex natura propria habet dissolvere, et consumere, et hujusmodi effectus: sed inquantum est instrumentum animae vegetabilis, generat carnem. Thomas semble avoir fluctué sur ce point. Dans Thomas d'Aquin, Summa contra Gentiles, II, 89.15, il écrit que l'action de la chaleur naturelle ne s'étend pas jusqu'à la production de la chair. Toutefois, il revient plus tard à la thèse initiale: voir la citation de Thomas d'Aquin, Quaestiones disputatae de anima ci-dessus, note 34, et De potentia, 3, 8, ad 13: (...) forma accidentalis agit in virtute formae substantialis quasi instrumentum eius; sicut etiam in II De anima calor ignis dicitur esse instrumentum virtutis nutritivae; et ideo non est inconveniens, si actio formae accidentalis ad formam substantialem terminetur.

37 Thomas d'Aquin, Summa contra Gentiles, II, 89.15: Cum igitur omnis virtus naturae activa comparetur ad Deum sicut instrumentum ad primum et principale agens, nihil prohibet in uno et eodem generato quod est homo, actionem naturae ad aliquid hominis terminari, et non ad totum quod fit actione Dei. Corpus igitur hominis formatur simul et virtute Dei quasi principalis agentis et primi, et etiam virtute seminis quasi agentis secundi: sed actio Dei producit animam humanam, quam virtus seminis producere non potest, sed disponit ad eam.

38 Réciproquement, il est important, comme nous le verrons dans la conclusion, de noter que même les causes instrumentales du premier type, celles dont l' action contribue directement à l' effet principal, ont aussi une action qui prépare l' action de la cause principale.

39 Thomas d'Aquin, Scriptum super Sententiis, IV, d. 1, q. 1, a. 4, 1.c.: (...) sciendum, quod actio instrumenti quandoque pertingit ad ultimam perfectionem, quam principale agens inducit, aliquando autem non. Semper tamen pertingit ad aliquid ultra id quod competit sibi secundum suam naturam, sive illud sit ultima forma, sive dispositio, alias non ageret ut instrumentum.

40 Thomas d'Aquin, Summa theologiae, III, 62.1, ad 2: Dicendum quod instrumentum habet duas actiones: unam instrumentalem secundum quam operatur non in virtute propria sed 
respond à sa forme et ses pouvoirs causaux propres, et celle que lui fait accomplir la cause principale, action qui va plus loin et qu' elle exécute seulement par le pouvoir de la cause principale ${ }^{41}$. La seconde ne remplace pas la première. La chaleur (ou plutôt l'élément feu, qui est l' agent, par la chaleur, qui est sa propriété) accomplit ce qu' elle fait naturellement par son propre eidos et pouvoir causal: décomposer des matériaux; mais, ce faisant, elle contribue de plus à ce qu' elle ne peut faire par elle-même: la fabrication de la chair par une âme végétative. De même, c' est en exécutant son action de base, couper du bois, qu'un outil de menuiserie contribue de plus à y introduire la forme d'un meuble ${ }^{42}$.

Nous pouvons appeler cela causalité instrumentale au sens fort, par opposition à celle qui résulte du troisième sens selon lequel une chose peut être cause de l'action d' une autre ${ }^{43}$. Certes, pour le sens 3 Thomas prend l'exemple d'un couteau, ce qui incite à voir déjà là un exemple de causalité instrumentale - et il est certes légitime de considérer un couteau comme un instrument. Toutefois, ce sens 3 admet des situations où il paraît difficile de considérer la cause seconde comme un instrument au sens strict. Si j' entaille un de mes doigts en coupant du pain, il est un peu tiré par les cheveux de parler du couteau comme d' un instrument à l' égard de cet effet-là; pourtant, la relation de cause motrice à cause mue reste exactement la même. Au contraire, le sens 4 ne correspond qu'à des cas où la cause seconde est un instrument au sens strict, parce que ce

in virtute principalis agentis; aliam autem habet actionem propriam quae competit ei secundum propriam formam.

41 Thomas d'Aquin, De potentia, 3.7.c.: Nec causa secunda potest in effectum causae primae per virtutem propriam, quamvis sit instrumentum causae primae respectu illius effectus. Instrumentum enim est causa quodammodo effectus principalis causae, non per formam vel virtutem propriam, sed in quantum participat aliquid de virtute principalis causae per motum eius. - Thomas d'Aquin, Summa theologiae, Ia IIae, 122.1, ad 1: Instrumentum non agit actionem agentis principalis propria virtute, sed virtute principalis agentis.

Thomas d'Aquin, Scriptum super Sententiis IV, d. 1, q. 1, a. 4.1.c.: (...) omne instrumentum agendo actionem naturalem, quae competit sibi inquantum est res quaedam, pertingit ad effectum qui competit sibi inquantum est instrumentum, sicut dolabrum dividendo suo acumine pertingit instrumentaliter ad formam scamni. - Thomas d'Aquin, Summa contra Gentiles, II, 21.7: Omne agens instrumentale exequitur actionem principalis agentis per aliquam actionem propriam et connaturalem sibi: sicut calor naturalis generat carnem dissolvendo et digerendo, et serra operatur ad perfectionem scamni secando. - Thomas d'Aquin, Summa theologiae, III, 62.1, ad 2: (...) securi competit scindere ratione suae acuitatis, facere autem lectum inquantum est instrumentum artis. Non autem perficit actionem instrumentalem nisi exercendo actionem propriam; scindendo enim facit lectum.

43 Thomas d'Aquin, De potentia, 3.7.c.: (...) quarto modo unum est causa actionis alterius, sicut principale agens est causa actionis instrumenti. Il faut remarquer que, dans ce texte, c' est seulement maintenant que Thomas introduit le terme d' instrument, alors que dans le sens 3 il ne parle pas d'instrumentalité (pas davantage que dans les sens 1 et 2). 
sens exige que la cause principale fasse faire à la cause subordonnée quelque chose qu'il n'est pas dans sa seule nature de faire. Si je me sers du couteau pour, simplement, couper, l' application que je fais de son pouvoir ne va pas audelà de ce que sa nature est capable d'accomplir. La nature du couteau est de trancher une fois qu'il est mis en mouvement, que ce soit du pain ou un doigt. Il faut simplement que son pouvoir soit actualisé par le mouvement de mon bras. Si maintenant je me sers de ce couteau pour graver, ce que le couteau fait va au-delà de ce que sa seule nature est capable d'accomplir par elle-même lorsqu' elle est mue. En fait, Thomas prend, pour le sens 4, l' exemple d'une doloire, qui serait l'instrument de la fabrication d'un meuble; mais pour un meilleur contraste entre sens 3 et 4 , je reprends celui d' un couteau, car le point important est la différence entre deux types d'utilisation d'un outil. Thomas ne donne pas deux fois le même exemple de causalité en changeant simplement d'outil; cela ne correspondrait pas à la progression des idées qu' il voulait mettre en œuvre dans ce passage. Le deuxième exemple d'utilisation d'un outil (pour $\mathrm{S}_{4}$ ), qui est spécifique de la causalité instrumentale au sens strict, va plus loin que le premier exemple (pour $\mathrm{S}_{3}$ ). Comme dans l'action de couper du pain, l'action du couteau est encore de trancher (ici, les fibres du bois), ce qui est son action propre; mais l' arrangement de ces différentes coupures dans le bois en figures bien déterminées (mots ou dessins) - ce qui est son action instrumentale au sens strict - relève d' une causalité supérieure, celle de l'esprit, dont l'action ne se borne pas à le mettre en mouvement. Le fait que les coupures soient disposées en un certain ordre n' est pas explicable causalement par le seul eidos du couteau. Par conséquent, non seulement il faut que son pouvoir soit actualisé par une force extérieure, mais il faut que s' ajoute à cela une causalité directrice qui est d' un autre ordre que la force mécanique requise pour l' action de couper, et qui est la cause principale de l'effet ${ }^{44}$. Cependant, c' est bien le couteau qui entaille le bois, pas l' esprit ni la main. Le dessin n' est pas explicable seulement par les propriétés de l'outil, mais il n' est pas non plus réductible à la seule action de l'agent principal. En somme, la causalité de l'esprit ne s'exerce que par l'intermédiaire du couteau, et celle du couteau ne s' exerce que sous la direction de l'esprit. Ces deux causalités se combinent

44 Thomas d'Aquin, De potentia, 3.7.c.: (...) dolabra non est causa rei artificiatae per formam vel virtutem propriam, sed per virtutem artificis a quo movetur et eam quoquomodo participat. Thomas d'Aquin, De unitate intellectus, 3, c.: actio moventis propria non attribuitur instrumento aut moto, sed magis e converso, actio instrumenti attribuitur principalimoventi: non enim potest dici quod serra disponat de artificio; potest tamen dici quod artifex secat, quod est opus serrae. 
donc en une seule action pour produire un seul effet ${ }^{45}$. Théodore de Régnon a commenté cela très éloquemment:

L'action est commune à l'agent principal et à l'instrument. L'action propre du ciseau, en vertu de sa forme, est de tailler la pierre; mais son action, comme instrument du sculpteur, est de faire une statue. Pas un trait de cette statue par où n'ait passé le ciseau. Donc, dans tous ses moindres détails, l'œuvre est effectuée par le ciseau, en même temps que par le sculpteur. La cause principale et la cause instrumentale produisent ensemble un seul et même effet. J' ajoute que cet effet ne résulte pas de deux actions différentes se rencontrant dans un même sujet. Il y a deux activités en exercice, mais le marbre ne subit qu'une seule passion, c'està-dire, une seule série de modifications. Or, l'action est corrélative de la passion; à une seule passion correspond une seule action. Donc le sculpteur et le ciseau exercent sur le marbre une unique et même action ${ }^{46}$.

En résumé, nous pouvons donc poser que:

(Déf.) Une entité $x$ est une cause instrumentale au sens fort ( $\left.\mathrm{S}_{4}\right)$ si et seulement si elle a simultanément deux actions: une action $\varphi$ qui résulte simplement de sa nature, et un autre type d'action, $\chi$, qu'elle ne peut effectuer d'elle-même, qui est due à sa subordination à une cause principale, et qui participe, directement ou en préparation, à la production de l'effet de cette cause principale.

Cette définition doit être complétée par trois clauses qui spécifient certains points essentiels:

(C1) Premièrement, l'action $\chi$ est une action commune à la cause principale et à la cause instrumentale ${ }^{47}$, et l' effet de cette action $\chi$ leur est commun

45 Thomas d'Aquin, Summa contra Gentiles, III, 66.3: Quando aliqua agentia diversa sub uno agente ordinantur, necesse est quod effectus qui ab eis communiter fit, sit eorum secundum quod uniuntur in participando motum et virtutem illius agentis: non enim plura faciunt unum nisi inquantum unum sunt.

46 Régnon 19o6, p. 482.

47 Thomas d'Aquin, Summa theologiae, III, 19.1, ad 2: Actio instrumenti, in quantum est instrumentum, non est alia ab actione principalis agentis. Potest tamen habere aliam actionem prout est res aliqua. Cf. Thomas d'Aquin, Summa theologiae Ia IIae, 14.3, ad 4: Agens principale et instrumentale sunt quasi una causa, cum unum agat per alterum. Toutefois, en Quaestiones disputatae De anima 2c., Thomas affirme que l' action de l'agent principal est distincte de celle de l'instrument: (...) non enim est possibile, si aliqua substantia ope- 
aussi, même s' il ne s' agit que de la préparation d' un sujet pour une action ultérieure de la seule cause principale ${ }^{48}$. L' action de la cause instrumentale en tant qu' instrumentale n' est pas séparable de celle de la cause principale. Toutefois, leur effet conjoint est plus proprement dit être l' effet de la cause principale, puisque sans elle la cause instrumentale ne pourrait le produire. L'action de la cause principale est prééminente. Elle agit «plus fortement» (vehementius) sur cet effet que la cause seconde, explique Thomas en reprenant une expression du Livre des causes ${ }^{49}$.

(C2) Deuxièmement, cette combinaison de pouvoir causaux en une seule action n'est possible que pour des causes qui appartiennent à des ordres différents, l' une supérieure, l' autre inférieure ${ }^{50}$. Quand les causes sont de même ordre, il y a autant d'actions que d'agents ${ }^{51}$. Cette combinaison de causalités en une seule action est en fait spécifique de la causalité instrumentale.

ratur aliquam operationem, quod illa operatio sit alterius substantiae ab ea diversa. Licet enim duarum substantiarum diversarum una possit alteri esse causa operandi ut principale agens instrumento, tamen actio principalis agentis non est actio instrumenti eadem secundum numerum, cum actio principalis agentis sit in movendo instrumentum; actio vero instrumenti in moveri a principali agente, et movere aliquid alterum. Mais étant donné qu' il s' attaque dans ce passage à la théorie averroïste de l'intellect possible, il y parle donc en fait de la causalité instrumentale au sens 3 du passage de De potentia 3.7 examiné plus haut, lorsqu' une substance simplement actualise le pouvoir causal d' une autre, ce qui est différent du sens 4 discuté ici.

48 Thomas d'Aquin, Summa contra Gentiles, III, 70.5: Sicut igitur non est inconveniens quod una actio producatur ex aliquo agente et eius virtute, ita non est inconveniens quod producatur idem effectus ab inferiori agente et Deo: ab utroque immediate, licet alio et alio modo.

49 Thomas d'Aquin, Summa contra Gentiles, II, 8.15: (...) causa agens prima agit in effectum causae secundae agentis vehementius quam etiam ipsa causa secunda; unde videmus quod effectus qui per instrumentum agitur a principali agente, magis proprie attribuitur principali agenti quam instrumento. Voir n. 19, ci-dessus. Voir aussi De potentia 3.7.c.: Quanto enim aliqua causa est altior, tanto est communior et efficacior, et quanto est efficacior, tanto profundius ingreditur in effectum (...).

50 Thomas d'Aquin, Summa contra Gentiles, II, 89.15: Quod vero tertio obiicitur, diversorum agentium actiones non terminari ad unum factum, intelligendum est de diversis agentibus non ordinatis. Si enim ordinata sint ad invicem, oportet eorum esse unum effectum (...). Thomas d'Aquin, Summa theologiae, I, 105.5, ad 2: (...) una actio non procedit a duobus agentibus unius ordinis; sed nihil prohibet quin una et eadem actio procedat a primo et secundo agente.

51 C'est l'un des arguments de Thomas pour réfuter le dualisme âme-corps: voir Thomas d'Aquin, Summa contra Gentiles, II, 57. Cf. Thomas d'Aquin, Summa theologiae, I, 52.3.c.: (...) impossibile est quod duae causae completae sint immediatae unius et eiusdem rei. Quod patet in omni genere causarum, una enim est forma proxima unius rei, et unum est proximum movens, licet possint esse plures motores remoti. Thomas sous-entend probablement ici que les deux causes totales seraient de même ordre; et c' est pourquoi elles ne peuvent être causes de la même chose. 
(C3) Troisièmement, la cause principale pénètre et agit dans le pouvoir de la cause subordonnée pour élever l'action de cette dernière au-dessus de ce qu' elle accomplit d'elle-même: par exemple, le «pouvoir» d'un artisan ou d'un artiste est reçu et participé par leur instrument ${ }^{52}$. Mais, alors que certaines causes inférieures agissent en vertu d'une forme que leur communique réellement et en propre la cause principale (ainsi, le métal du radiateur chauffe parce que l' eau du circuit lui communique sa chaleur, qui devient une forme accidentelle du métal), en revanche le pouvoir d'exécuter l'action $\chi$, donné par la cause principale à la cause instrumentale, n' est reçu en celle-ci que sur un mode dit «intentionnel» (par opposition au mode plein et entier selon lequel une forme est normalement reçue dans un sujet), c' est-à-dire un mode incomplet et transitoire, semblable au mode selon lequel les espèces sensibles qui transmettent les couleurs des choses sont présentes dans l'air (elles y sont effectivement, sans toutefois être capables de colorer l' air, et ne sont donc pas vues $)^{53}$. C' est de cette manière que l' art d'un peintre se matérialise sur une toile par l'intermédiaire de son pinceau. Son savoir-faire anime son pinceau, pour ainsi dire ${ }^{54}$. Pour Thomas, cela signifie qu'il y réside sur un mode intentionnel - il ne pourrait évidemment y être reçu sur un mode ontologique normal,

52 Thomas d'Aquin, Scriptum super Sententiis, IV, d. 1, q. 1, a. 1, 4, ad 1: (...) sacramentum est causa et signum. Est quidem causa instrumentalis; et ideo virtus agentis principalis occulte in ipso operatur, sicut virtus artis vel artificis in serra. Thomas d' Aquin, Scriptum super Sententiis, IV, d. 1, q. 1, a. 4, 3, c.: (...) instrumentum praedicto modo virtutem non accipit nisi secundum quod principali agenti continuatur, ut virtus ejus quodammodo in instrumentum transfundatur.

53 Thomas d'Aquin, Scriptum super Sententiis, IV, d. 1, q. 1, a. 4, 2, c.: Agens enim principale agit secundum exigentiam suae formae; et ideo virtus activa in ipso est aliqua forma vel qualitas habens completum esse in natura. Instrumentum autem agit ut motum ab alio; et ideo competit sibi virtus proportionata motui: motus autem non est ens completum sed est via in ens quasi medium quid inter potentiam puram et actum purum, ut dicitur in III Physicorum. Et ideo virtus instrumenti inquantum hujusmodi, secundum quod agit ad effectum ultra id quod competit sibi secundum suam naturam, non est ens completum habens esse fixum in natura, sed quoddam ens incompletum, sicut est virtus immutandi visum in aere, inquantum est instrumentum motum ab exteriori visibili; et hujusmodi entia consueverunt intentiones nominari, et habent aliquid simile cum ente, quod est in anima quod est ens diminutum, ut dicitur in vi Metaphysicorum. Thomas d'Aquin, Scriptum super Sententiis, IV, d. 1, q. 1, a. 4,4 : (...) in re corporali non potest esse virtus spiritualis secundum esse completum; potest tamen ibi esse per modum intentionis, sicut in instrumentis motis ab artifice est virtus artis (...).

54 Cela est également par opposition à une cause qui est purement et simplement mue par l'agent supérieur, comme l' est le couteau qui coupe du pain (sens 3), auquel cas l'agent supérieur n'imprime aucune forme dans la cause inférieure. 
comme il l' est dans l'âme de l'artiste (en tant qu'habitus) ou comme une couleur l' est sur la toile.

Il s' ensuit trois spécifications importantes, que nous pouvons aussi compter comme des clauses qui précisent ce qu' est la causalité instrumentale au sens fort:

(C4) La première est que, en plus d'avoir ce statut ontologique diminué, incomplet, le pouvoir de faire $\chi$ ne réside que temporairement dans la cause instrumentale, pour aussi longtemps que la cause principale se la subordonne et agit sur elle ${ }^{55}$. Lorsque cette influence cesse, la cause instrumentale cesse de pouvoir exécuter l' action $\chi$. Le pinceau ne garde pas le pouvoir de réaliser une œuvre d' art. La forme de l' effet ne se trouve en lui qu' «en flux» et sur un mode transitoire, contrairement aux espèces cognitives intentionnelles ${ }^{56}$.

(C5) La deuxième est que, puisque la forme fondement du pouvoir causal ne réside pas sur un mode réel dans la cause instrumentale, l' effet ne porte pas la ressemblance de cette cause instrumentale, mais porte la ressemblance de la cause principale, en laquelle la forme se trouve réellement ${ }^{57}$.

(C6) Enfin, pour la même raison l'effet peut être plus parfait, non pas que la cause principale, mais que la cause instrumentale ${ }^{58}$. La forme de l'effet ne se trouve pas dans l'instrument selon toute la ratio de sa nature, mais ne s'y trouve que sur un mode déficient ${ }^{59}$.

55 Voir Thomas d'Aquin, Scriptum super Sententiis, IV, d. 1, q. 1, a. 4, 3, c., cité ci-dessus n. $5^{2 .}$ Voir aussi De potentia 3, 7, ad 7 et 3, 11, ad 14; Scriptum super Sententiis, IV, d. 1, q. 1, a. 4, 4.

56 Thomas d'Aquin, Scriptum super Sententiis, IV, d. 1, q. 1, a. 4, 4, c.: Tertio, quia non est [forma] in [instrumento] per modum intentionis quiescentis, sicut sunt intentiones rerum in anima, sed per modum intentionis fluentis duplici fluxu; quorum unus est de potentia in actum, sicut etiam in mobili est forma, quae est terminus motus, dum movetur ut fluens de potentia in actum, et inter haec cadit medium motus, cujus virtute instrumentum agit; alius de agente in patiens, inter quae cadit medium instrumentum, prout unum est movens, et alterum motum.

57 Thomas d'Aquin, Scriptum super Sententiis, IV, d. 1, q. 5, a. 2, 2, c.: Agens autem principale, cum agat sibi simile, oportet quod habeat formam, quam inducit per suam actionem in agentibus univocis, vel aliquam nobiliorem in agentibus non univocis. Sed agens instrumentale non oportet quod habeat formam quam inducit ut disponentem ipsum, sed solum permodum intentionis, sicut de forma scamni in serra patet. Thomas d'Aquin, Summa theologiae, III, 62.1: Duplex est causa agens, principalis et instrumentalis. Principalis quidem operatur per virtutem suae formae, cui assimilatur effectus, sicut ignis suo calore calefacit. Causa vero instrumentalis non agit per virtutem suae formae, sed solum per motum quo movetur a principali agente. Unde effectus non assimilatur securi, sed arti quae est in mente artificis.

$5^{8}$ Thomas d' Aquin, Summa theologiae, III, 79.2, ad 3: Nihil prohibet causam instrumentalem producere potiorem effectum.

59 Thomas d'Aquin, Scriptum super Sententiis, IV, d. 1, q. 1, a. 4, 4.c.: (...) in instrumento non 
Autrement dit, la causalité instrumentale n' obéit pas à ces deux lois de la causalité univoque ordinaire: un agent produit quelque chose qui lui ressemble (agens agit sibi simile), et la cause est plus noble que l'effet (causa nobilior effectu est).

\subsection{Application à la causalité divine}

Nous pouvons maintenant appliquer cette théorie de la causalité instrumentale à la question du concours de Dieu aux actions des créatures. Dieu peut être dit cause de l'action de toutes les autres causes en chacun des sens qui viennent d'être définis; seul le quatrième, toutefois, correspond à ce qui est nécessaire pour rendre compte de la donation d' existence.

$(\mathrm{S} 1)$ Si une chose cause l'action d'une autre quand elle donne à cette autre chose son pouvoir d'action, il est clair que cela est le cas pour Dieu à l'égard des créatures. Mais cela ne concerne pas la production d'existence, puisque justement Dieu ne donne pas ce pouvoir causal en propre aux créatures.

(S2) Si une chose cause l'action d'une autre quand elle préserve son pouvoir causal contre la corruption, c'est de nouveau le cas pour Dieu à l'égard des créatures. Non seulement il a donné aux choses leur pouvoir d'agir, mais il maintient leur pouvoir dans l' existence en les empêchant de retomber dans le néant - c'est là l'importante thèse de la conservation des créatures par Dieu (que je n' examine pas ici, comme je l' ai dit). Mais pour la même raison que précédemment, cela ne concerne pas la production d'existence, que les créatures n' ont précisément pas le pouvoir d' accomplir.

(S3) Si une chose cause l' action d' une autre lorsqu' elle «applique» le pouvoir de celle-ci, c' est-à-dire la meut à agir, cela est encore une fois le cas pour Dieu à l'égard des créatures. Aucune des créatures ne se meut et n' agit sans être mue. Il leur faut un premier moteur, non mû. Cette cause première est en ce sens aussi la cause de toutes les actions de toutes les choses de la nature ${ }^{60}$. Dieu n' est pas seulement la cause des causes secondes, il est ultimement ce qui «applique» leur pouvoir à leurs effets ${ }^{61}$. Par la succession continue des actua-

est forma effectus secundum completam rationem speciei, sicut est in effectu jam completo, et in causa univoca (...).

6o Thomas d'Aquin, De potentia, 3.7.c.: Et quia natura inferiora agens non agit nisi mota eo quod huiusmodi corpora inferiora sunt alterantia alterata, caelum autem est alterans non alteratum et tamen non est movens nisi motum, et hoc non cessat quousque perveniatur ad Deum, sequitur de necessitate quod Deus sit causa actionis cuiuslibet reinaturalis ut movens et applicans virtutem ad agendum.

61 Cf. Thomas d' Aquin, Summa contra Gentiles, III, 67.4: Quicquid applicat virtutem activam ad agendum, dicitur esse causa illius actionis: artifex enim applicans virtutem rei naturalis ad aliquam actionem, dicitur esse causa illius actionis, sicut coquus decoctionis, quae est per 
lisations: mouvements célestes, mouvements sublunaires, etc., Dieu est cause de tout ce qui se fait dans l'univers. Le fabricant du couteau et l' aiguiseur qui le garde tranchant sont en un sens (sens 1 et 2) causes que le couteau coupe; mais Dieu, qui est comme le fabricant et l' aiguiseur (création et conservation), est de plus comme l'utilisateur du couteau (application du pouvoir de ce dernier, sens 3). Néanmoins, encore une fois, et toujours pour la même raison, cela ne s'applique pas à la donation d' existence.

(S4) La communication de l'existence aux effets a ceci de particulier que, d'une part, le pouvoir correspondant appartient à Dieu exclusivement, mais que, d'autre part, l' action des créatures dans cette donation d' existence n' est pas superflue et doit être inclue de droit dans le processus. Le quatrième des sens en lesquels une chose cause l'action d' une autre, c'est-à-dire la causalité instrumentale au sens fort, correspond le mieux à ce schéma. De par sa structure même, la causation instrumentale proprement dite réserve à la cause principale un pouvoir qui cependant opère à travers l'instrument, de sorte que ce dernier contribue à un effet qui est au-delà de ce qu'il peut faire par luimême. La causation instrumentale répond de plus aux réquisits qui ont été formulés plus haut concernant la manière dont la causalité de Dieu doit se combiner à celle d' une créature:

- Les deux causes doivent être réellement causes $(\mathrm{R} 1)$, être toutes deux nécessaires $\left(R_{3}\right)$, et être toutes deux causes totales de cet effet $\left(R_{5}\right)$, ce qui est satisfait par la définition même de la causalité instrumentale;

- Elles doivent être hiérarchiquement ordonnées (R4) - ce qui est satisfait par la clause $\mathrm{C}_{2}$;

- Elles doivent contribuer à une même action et un même effet $(\mathrm{R} 2)$, ce qui est satisfait par la clause $\mathrm{C}$.

Tel est donc le modèle qu' adopte Thomas pour compléter l' architecture causale du Livre des causes et décrire le concours divin aux actions des créatures en ce qui concerne la donation d' existence ${ }^{62}$. Utiliser un modèle, toutefois,

ignem. Sed omnis applicatio virtutis ad operationem est principaliter et primo a Deo. Applicantur enim virtutes operativae ad proprias operationes per aliquem motum vel corporis, vel animae. Primum autem principium utriusque motus est Deus. Est enim primum movens omnino immobile, ut supra ostensum est.

62 Mancha 2012, p. 350-351, argues avec Suárez (Disputatio metaphysica XXII, section 2, par. 52 , p. 824) que Thomas ne parle de cette quatrième manière d'être cause de l' activité d'une autre cause que dans De Pot. 3.7.c. Suárez vas jusqu' à dire que dans Summa theologiae I, 105.5, c. Thomas rétracte implicitement cette doctrine en la passant sous silence et n'utilise le modèle instrumental que dans le sens 3 du De potentia, c' est-à-dire celui de «mettre en mouvement» les causes secondes. L'argument me paraît faible. Premièrement, il résulte de ce que Suárez entend purger le concurrentisme de tout élément néo- 
n' est pas fournir une preuve. Je ne prétends donc pas que Thomas démontre la validité de l'étiologie proclusienne au moyen de la causalité instrumentale. Mais, avec ce modèle, élaboré à partir d'une observation pour ainsi dire phénoménologique de la relation d'instrumentalité, il tente d'élucider et de rendre plus familière l' intrication des actions causales sur laquelle repose l'étiologie proclusienne. D' où le fait que Thomas associe souvent une référence au De causis, explicite ou implicite, à une analyse en termes de causalité instrumentale, comme s'il appuyait cette dernière par l'autorité du De causis, ou aussi bien expliquait cette dernière par la première ${ }^{63}$.

Revenons en quelque détail sur certains points pour encore mieux voir pourquoi et comment le modèle de l'instrumentalité peut aider à comprendre la relation entre causalité divine et causalité des créatures. Seul Dieu a le pouvoir de conférer l'être, comme nous l'avons vu dans la première partie. Néanmoins, l' action des créatures aboutit aussi à amener un effet à l' existence, que ce soit une nouvelle substance, dans les processus de génération, ou un nou-

platonicien (la participation de la cause instrumentale au pouvoir de la cause principale). En fait, il entend même se débarrasser de la notion d'instrumentalité dans ce contexte (voir ibid., par. 53), car, une fois rejetée la pénétration de l'action créaturelle par l'action divine, le modèle instrumental devient défectueux: d' une part Dieu n' est que cause lointaine car la cause seconde devient un intermédiaire entre Dieu et l' effet (ibid., section 1, par. 19), et d'autre part, instrument inerte, la cause seconde est par trop dépouillée de sa causalité propre (ibid., section 2, par. 25, 31-40). Deuxièmement, les questions De potentia ne précède que de peu la première partie de la Somme: probablement 1265-1266 pour les premières, 1266-1268 pour la seconde, toutes composées pendant le séjour de Thomas à Rome (voir Torrell 1996, p. 333 et 335); cela laisse peu de temps pour une évolution de la pensée de Thomas. Troisièmement, comme on sait la Somme est un abrégé de théologie, les questions disputées sont généralement plus détaillées, et c' est donc dans le De potentia qu'il faut chercher la présentation la plus élaborée de la théorie de Thomas, l' argument de Suárez ne valant que ce que valent les arguments $e$ silentio. Quatrièmement, le sens 3 ne suffit pas à assurer ce que Thomas veut démontrer, à savoir que c'est Dieu seul qui donne l'être, même s'il le fait à travers l' action de créatures. Une cause seconde au sens 3 doit certes être mue par une cause principale; mais cela n' empêche pas qu' elle seule effectue l'action concernée: c' est le couteau qui tranche, non pas le bras, ou c' est le feu qui cuit, non pas le cuisinier directement (cf. Summa contra Gentiles, III, 67.4).

63 Ainsi, dans le passage crucial de De potentia 3.7, c.: (...) et hoc modo etiam oportet dicere, quod Deus est causa omnis actionis rei naturalis. Quanto enim aliqua causa est altior, tanto est communior et efficacior, et quanto est efficacior, tanto profundius ingreditur in effectum (...) unde etiam, ut dicitur in Lib. de causis, intelligentia non dat esse, nisi prout est in ea virtus divina. Sic ergo Deus est causa omnis actionis, prout quodlibet agens est instrumentum divinae virtutis operantis. (...) nam virtus inferior non coniungitur effectui nisi per virtutem superioris; unde dicitur in Libro de causis quod virtus causae primae prius agit in causatum, et vehementius ingreditur in ipsum. Voir aussi Summa contra Gentiles II, 8.15, cité ci-dessus n. 49 . 
vel accident, dans les autres types de changement. Les créatures sont en effet pour Thomas des causes réelles, non pas occasionnelles, et sont de surcroît des causes totales. Une cause réelle et totale actualise une forme, substantielle ou accidentelle, dans un sujet. Or, actualiser une forme revient à actualiser un esse, substantiel ou accidentel: un composé (soit forme substantielle / matière, soit forme accidentelle / substance) vient en effet à l'existence par le fait qu'une forme est actualisée ${ }^{64}$. Les agents créés produisent donc toujours de l' existant, bien que l'existence ne vienne pas d'eux. C'est là la tension qui impose le recours à la causalité instrumentale. La seule possibilité pour concilier l' action causale des créatures avec celle de Dieu est que la première soit instrumentale à l' égard de la donation d'être, qui vient de Dieu comme cause principale. Conformément à la théorie de l'instrumentalité (voir «Déf.» plus haut), cela ne signifie pas que Dieu délègue à des créatures le pouvoir de créer, mais que l'action créatrice de Dieu passe par l' activité causale naturelle $\varphi$ des créatures (qui par elle-même est incapable de faire exister l' effet), et leur fait exécuter une action $\chi$ qui aboutit à la venue d' un effet à l' existence. Dans les termes du De causis: la cause première agit sur l' effet au sein même de l' action de la cause prochaine. Une cause seconde est donc toujours cause d'un exister, mais elle n'en est pas la cause principale. Dieu est en ce sens la cause de toute action des créatures, car l' existence est en fait donnée par lui aux effets, et tout autre agent est cause instrumentale de la donation d'existence ${ }^{65}$.

Si l'on regarde cet agencement en sens inverse, c' est-à-dire du point de vue de l' effet, on remarque dans ce dernier différentes strates de propriétés reçues, qui chacune correspondent à l'une des causes qui co-agissent, en vertu de ce principe que nous avons déjà rencontré et que Thomas tient pour axiomatique, à savoir que l' ordre entre les effets correspond à l' ordre entre les causes ${ }^{66}$. Ainsi, dans l'exemple proposé pour le sens 4 dans De Potentia 3.7, on doit distinguer

64 Thomas d'Aquin, Scriptum super Sententiis, I, d. 17, q. 1, a. 1: Constat enim quod omne esse a forma aliqua inhaerente est, sicut esse album ab albedine, et esse substantiale a forma substantiali. Voir ci-dessus, n. 8.

65 Thomas d'Aquin, De potentia, 3.4.c.: (...) quaecumque alia causa dat esse, hoc habet in quantum est in ea virtus et operatio primae causae, et non per propriam virtutem; sicut et instrumentum efficit actionem instrumentalem non per virtutem propriae naturae, sed per virtutem moventis; sicut calor naturalis per virtutem animae generat carnem vivam, per virtutem autem propriae naturae solummodo calefacit et dissolvit. Thomas d' Aquin, De potentia, 3.7, c.: Sic ergo Deus est causa omnis actionis, prout quodlibet agens est instrumentum divinae virtutis operantis. Thomas d'Aquin, Summa contra Gentiles, III, 67.1: Omne enim operans est aliquo modo causa essendi, vel secundum esse substantiale, vel accidentale. Nihil autem est causa essendi nisi inquantum agit in virtute Dei, ut ostensum est. Omne igitur operans operatur per virtutem Dei.

66 Thomas d'Aquin, De potentia, 3.7.c.: Sed ulterius invenimus, secundum ordinem causarum, 
dans l' effet la strate propre de la causalité du couteau, qui est le tranchement des fibres, et la strate propre de la causalité de l'esprit, qui est l' agencement de ces coupures. De même, il y a dans les effets de processus naturels des strates qu' une cause seconde aurait été incapable de produire par elle-même. À cette superposition de propriétés ou de strates (être, matérialité, essence spécifique, caractéristiques individuelles) ${ }^{67}$, correspond une hiérarchie de causalités emboîtées les unes dans les autres. Un individu, en tant qu'individu, ne peut communiquer à son effet que des caractéristiques individuelles ${ }^{68}$. La nature spécifique qu' il a transmise a été causée non par lui en tant qu' individu, mais par une cause plus générale à travers lui, à savoir les corps célestes, qui agissent sur les éléments dans les processus de génération - autrement dit, un être vivant n' en engendre un autre de même nature que par la vertu des cieux, dont il est cause instrumentale ${ }^{69}$. La couche la plus fondamentale et la plus commune est, nous le savons, l' exister, qui ne peut être produit que par la cause première. Ce ne sont ni l'individu ni les cieux seuls qui ont pu causer le soubassement de la nature spécifique, l'être simpliciter ${ }^{70}$. Nous retrouvons donc le principe proclusien: plus une cause est élevée, plus son action est étendue et efficace; et plus elle est efficace, plus elle pénètre profondément dans l'effet et le tire d'un plus grand état de potentialité. Inversement, plus l' effet est universel et intérieur aux choses, plus sa cause doit être élevée. Cette ultime stratification correspond bien sûr à la distinction entre causalité par création et causalité par information que l' on trouve dans le Livre des causes ${ }^{71}$. La cau-

esse ordinem effectuum, quod necesse est propter similitudinem effectus et causae. Voir cidessus, p. 308 et n. 16, l' argument de Thomas d'Aquin, De potentia, 3.4.c.

67 Thomas d'Aquin, De potentia, 3.7.c.: In qualibet autem re naturali invenimus quod est ens et quod est res naturalis, et quod est talis vel talis naturae. Quorum primum est commune omnibus entibus; secundum omnibus rebus naturalibus; tertium in una specie; et quartum, si addamus accidentia, est proprium huic individuo.

68 Thomas d'Aquin, Summa theologiae, I, 45.5, c.: Non enim hic homo potest esse causa naturae humanae absolute, quia sic esset causa sui ipsius, sed est causa quod natura humana sit in hoc homine generato. Et sic praesupponit in sua actione determinatam materiam per quam est hic homo.

69 Thomas d'Aquin, De potentia, 3.7, c.: Hoc ergo individuum agendo non potest constituere aliud in simili specie nisi prout est instrumentum illius causae, quae respicit totam speciem et ulterius totum esse naturae inferioris. Et propter hoc nihil agit ad speciem in istis inferioribus nisi per virtutem corporis caelestis (...). Voir les autres textes rassemblés par Litt 1963, p. 165-172.

70 Thomas d'Aquin, De potentia, 3.7.c.: (...) nihil agit ad speciem in istis inferioribus nisi per virtutem corporis caelestis, nec aliquid agit ad esse nisi per virtutem Dei. Ipsum enim esse est communissimus effectus primus et intimior omnibus aliis effectibus; et ideo soli Deo competit secundum virtutem propriam talis effectus (...).

71 Voir ci-dessus p. 305 et n. 6. Naturellement, on trouve aussi la même distinction chez Avicenne. 
salité qui ajoute à l'être est celle des causes secondes, qui agissent par information, sur la base d'un effet de la seule cause première (l' exister). L' exister est donné par création, l'être-tel ou tel, comme le «vivre» et toute autre propriété de ce genre, est donné par information ${ }^{72}$. L' effet propre de l' action divine est l'existence, l' effet propre des causes secondes est que ce qui existe soit de telle ou telle nature, c' est-à-dire est une détermination de l'esse à être existence de telle ou telle sorte, et à être un individu avec telles ou telles caractéristiques individuelles ${ }^{73}$.

Mais de même que l'individu est cause instrumentale à l'égard de la causalité propre des corps célestes, l'individu et les corps célestes sont causes instrumentales à l'égard de la causalité réservée à Dieu. En d' autres termes, la donation de l'existence ne peut venir que de la cause première agissant présentement sur l'effet, mais cette donation s' effectue au travers des causes subordonnées - non pas en les investissant d'un pouvoir que la cause première n'a de ce fait plus à exercer, mais en se subordonnant leur action et agissant par elle en vue d'un effet supérieur ${ }^{74}$. C' est bien une créature matérielle qui

72 Thomas d'Aquin, De potentia, 3.1, c: (...) in nulla re naturali includuntur actus et perfectiones omnium eorum quae sunt in actu; sed quaelibet illarum habet actum determinatum ad unum genus et ad unam speciem; et inde est quod nulla earum est activa entis secundum quod est ens, sed eius entis secundum quod est hoc ens, determinatum in hac vel illa specie: nam agens agit sibi simile. Et ideo agens naturale non producit simpliciter ens, sed ens praeexistens et determinatum ad hoc vel ad aliud (...). Et inde est quod in Libro de causis dicitur, quod esse eius est per creationem, vivere vero, et caetera huiusmodi, per informationem. Causalitates enim entis absolute reducuntur in primam causam universalem; causalitas vero aliorum quae ad esse superadduntur; vel quibus esse specificatur, pertinet ad causas secundas, quae agunt per informationem, quasi supposito effectu causae universalis; et inde etiam est quod nulla res dat esse, nisi in quantum est in ea participatio divinae virtutis. Propter quod etiam dicitur in Libro de causis, quod anima nobilis habet operationem divinam in quantum dat esse.

73 Thomas d'Aquin, Summa contra Gentiles, III, 66.6: Primum autem in omnibus effectibus est esse: nam omnia alia sunt quaedam determinationes ipsius. Igitur esse est proprius effectus primi agentis, et omnia alia agunt ipsum inquantum agunt in virtute primi agentis. Secunda autem agentia, quae sunt quasi particulantes et determinantes actionem primi agentis, agunt sicut proprios effectus alias perfectiones, quae determinant esse. Voir aussi Summa contra Gentiles, II.21: Alia vero agentia non sunt causa essendi simpliciter, sed causa essendi hoc, ut hominem vel album. Naturellement, ce que les causes secondes sont et donnent, et leur action même, leur viennent aussi de la cause première; mais c' est là, comme je l' ai dit plus haut (n. 9) un autre aspect de la théorie du concours. Quand donc je parle de l' effet propre des causes secondes, il s' agit de ce qu' elles sont capables de faire de par leur nature, étant entendu que cette nature et leur possibilité d' agir sont reconduites aussi à la cause première par une analyse ultérieure.

74 Thomas d'Aquin, De potentia, 5.1, ad 5: Si autem ponamus formas substantiales educi de potentia materiae, secundum sententiam Aristotelis, agentia naturalia non solum erunt cau- 
a produit une autre créature matérielle: tel homme a engendré tel homme. Le géniteur ne se borne pas à disposer la matière pour qu' ensuite Dieu seul donne l' existence à l' effet. Ce serait réduire la causalité naturelle de la créature à une causalité occasionnelle à l' égard de la donation d' existence. Bien plutôt, Dieu donne l'existence par l'intermédiaire de la créature. C' est l'individu qui communique l' existence à un autre individu - mais il ne le fait pas de lui-même ${ }^{75}$. Il faut en fait que les diverses causalités qui se sont exercées se soient superposées à celle qui est propre à l'individu et lui aient fait produire ce qu' à lui seul il ne pouvait produire. La cause seconde n' est qu' un instrument, mais c' est bien elle qui effectue l' action sous l'impulsion de la cause première ${ }^{76}$. L'instrument est donc « d' une certaine manière» cause de l' effet de la cause première (c' est-àdire l' existence). Il l' est non pas en vertu de sa forme et de son pouvoir propres, mais parce qu' il participe du pouvoir de la cause principale en étant agi par elle.

Autrement dit, la distinction de strates dans l' effet n' indique pas une pluralité d'actions parallèles. Comme je l' ai souligné, l' effet est entièrement produit à la fois par Dieu et par la créature (totus ab utroque $)^{77}$. L'agent principal et sa cause instrumentale contribuent conjointement au même et unique processus qui engendre un unique effet. Comme nous l'avons vu aussi, l'un ne produit pas une partie de l'effet et l' autre une autre partie. Bien sûr, leurs modalités d'action sont différentes; mais une substance est engendrée comme un tout, à la fois par Dieu et la créature. Dans un être vivant, le vivre et l'être sont en fait la même chose, car pour un vivant, exister c' est vivre (vivere viventibus est esse $)^{78}$. Il n'y a pas deux actes distincts, vivre d'un côté, et exister de

sae dispositionum materiae, sed etiam formarum substantialium; quantum ad hoc dumtaxat quod de potentia educuntur in actum, ut dictum est, et per consequens sunt essendi principia quantum ad inchoationem ad esse, et non quantum ad ipsum esse absolute.

Reprenons une phrase déjà citée de De potentia, 3.7, c., mais en l' accentuant autrement (je souligne): (...) nihil agit ad speciem in istis inferioribus nisi per virtutem corporis caelestis, nec aliquid agit ad esse nisi per virtutem Dei. (...) unde etiam, ut dicitur in Libro de causis, intelligentia non dat esse, nisi prout est in ea virtus divina. Voir aussi Summa contra Gentiles, III, 66.3: Cum igitur esse sit communis effectus omnium agentium (nam omne agens facit esse actu), oportet quod hunc effectum producunt inquantum ordinantur sub primo agente, et agunt in virtute ipsius.

76 Thomas d'Aquin, De potentia, 3.4, c.: Nam actio alicuius, etiamsi sit eius ut instrumenti, oportet ut ab eius potentia egrediatur.

77 Thomas d'Aquin, Summa contra Gentiles, III, 70.7: Patet etiam quod non sic idem effectus causae naturali et divinae virtuti attribuitur quasi partim a Deo, et partim a naturali agente fiat, sed totus ab utroque secundum alium modum: sicut idem effectus totus attribuitur instrumento, et principali agenti etiam totus.

78 Aristote, De anima II.4, 415b13. Thomas d'Aquin, Sententia Libri de anima I, 14.11: Unde et vivere dupliciter accipitur. Uno modo accipitur vivere, quod est esse viventis, sicut dicit Philo- 
l'autre, donnés indépendamment l'un de l' autre. La cause prochaine donne la vie parce qu' elle éduit la forme substantielle de la matière (sauf dans le cas de l'âme humaine $)^{79}$, et du même coup elle transmet l' existence ${ }^{80}$. La substance produite est donc «immédiatement et tout entière $(t o t a)$ » effet de Dieu, mais aussi «immédiatement et tout entière » effet de causes secondes. Dieu seul lui donne d' «être vivante» sous l' aspect de l'être, qui lui est commun avec toute autre créature - mais il le fait par les causes secondes -, et les causes secondes lui donnent en propre d' « être vivante» sous l' aspect de vivant, qui la distingue d'autres créatures. Les causes secondes spécialisent, pour ainsi dire, l'action de la cause première. Mais par là même, elles donnent aussi l' existence; non pas d'elles-mêmes, comme si elles étaient investies de ce pouvoir, mais dans la mesure où la cause première l'exerce au sein même de leur activité de causes secondes.

Il importe de préciser que cette action de la cause première n' est pas une sorte de force mystérieuse qui s'empare des causes subordonnées. Thomas rejette la théorie d'Avicebron, à savoir qu' aucune substance corporelle n' agit mais qu' un pouvoir spirituel pénètre les corps et agit en eux, et qu'un corps est d'autant plus actif qu'il est plus pur et subtil et peut être pénétré par cette force ${ }^{81}$. Pour Thomas, le pouvoir propre de la créature est suffisant pour produire dans l' effet la strate qui est de son ordre ${ }^{82}$. Mais, en même temps, la causalité supérieure agit au travers de la nature de la cause seconde. Le pouvoir naturel qu' une chose a reçu est dans cette chose comme une forme qui a un être établi et stable; en revanche, la causalité divine qui agit dans l' action de

sophus, quod vivere est esse viventibus. Alio modo vivere est operatio. Cf. Thomas d'Aquin, Scriptum super Sententiis, I, d. 8, q. 5, a. 3, ad 3: (...) uno modo vivere est ipsum esse viventis, sicut dicit philosophus: vivere viventibus est esse; et hoc modo anima immediate facit vivere quamlibet partem corporis, inquantum est ejus forma (...). Thomas d' Aquin, Scriptum super Sententiis, IV, d. 49, q. 1, a. 2, 3.c.: (...) vita dicitur dupliciter. Uno modo ipsum esse viventis; quia, ut in II De anima dicitur, vivere viventibus est esse. Ex hoc autem dicitur aliquid vivens quod potest seipsum movere secundum aliquam actionem (...).

79 Voir Thomas d'Aquin, Summa contra Gentiles, II, 89.15: Cum igitur omnis virtus naturae activa comparetur ad Deum sicut instrumentum ad primum et principale agens, nihil prohibet in uno et eodem generato quod est homo, actionem naturae ad aliquid hominis terminari, et non ad totum quod fit actione Dei. Corpus igitur hominis formatur simul et virtute Dei quasi principalis agentis et primi, et etiam virtute seminis quasi agentis secundi; sed actio Dei producit animam humanam, quam virtus seminis producere non potest, sed disponit ad eam.

8o Thomas d'Aquin, Sententia Libri de anima, II, 7.11: (...) anima viventibus est causa essendi; per animam enim vivunt, et ipsum vivere est esse eorum (...).

81 Thomas d'Aquin, De potentia, 3.7.c.

82 Thomas d'Aquin, De potentia, 3.7, ad 1: (...) virtus activa et passiva rei naturalis sufficiunt ad agendum in ordine suo. 
cette chose est reçue sur un mode d' être intentionnel, incomplet, comme l'être qu' ont les couleurs dans l'air, ou le pouvoir de l'artisan dans son instrument (voir clause $\mathrm{C}_{3}$ plus haut) ${ }^{83}$. Une cause seconde peut conférer d' elle-même sa caractéristique propre parce que celle-ci réside en elle d'une manière stable, mais elle ne peut pas conférer par elle-même l'existence qu' elle reçoit de la cause première et qu'elle transmet en tant qu'instrument (clause $\mathrm{C}_{4}$ ). A ce titre, elle contribue à produire un effet qui est supérieur à ce qu' elle peut de sa propre nature, comme toutes les causes instrumentales (clause C6). Et, sous l' aspect de l'être, l' effet produit imite davantage (mais avec déficience) Dieu, qui est être par essence, qu' aucune des causes subordonnées (clause $\mathrm{C}_{5}$ ).

Le fait que l'activité de la cause principale s'exerce à travers une cause seconde n'empêche pas que son activité soit, à l'égard de l'effet, aussi immédiate que celle de la cause seconde. Cela s'applique déjà à la causalité instrumentale au sens large (sens 3, simple application du pouvoir de la cause seconde): l'utilisateur du couteau est cause immédiate de l'action de couper, même s'il coupe par l'intermédiaire du couteau. Une cause principale éloignée, au contraire, est une cause qui n' agit pas en même temps que la cause seconde: si je lance mon couteau sur une cible, je suis cause éloignée de l' action de couper (non pas parce que je suis loin de la cible, mais parce que je n' agis plus sur le couteau au moment où il coupe). Mais si la cause principale agit en même temps que la cause seconde sur l'effet, elle aussi est cause immédiate. Le nombre d'instruments, c' est-à-dire d'intermédiaires, n'y change rien: si je manie mon couteau par l' intermédiaire d'un bâton auquel il est attaché, je suis tout de même cause immédiate de l' action de couper. C' est pourquoi, bien que Dieu soit cause initiale d'une série causale (les cieux, les créatures ici-bas, etc.), il est cause immédiate du dernier effet dans cette série ${ }^{84}$. A fortiori, dans la causalité instrumentale au sens fort, Dieu est encore davantage cause immédiate, puisqu' il est cause que l' existence soit donnée à l' effet. Si nous considérons les

83 Thomas d'Aquin, De potentia, 3.7, ad 7: (...) virtus naturalis quae est rebus naturalibus in sua institutione collata, inest eis ut quaedam forma habens esse ratum et firmum in natura. Sed id quod a Deo fit in re naturali, quo actualiter agat, est ut intentio sola, habens esse quoddam incompletum, per modum quo colores sunt in aere, et virtus artis in instrumento artificis. Sicut ergo securi per artem dari potuit acumen, ut esset forma in ea permanens, non autem dari ei potuit quod vis artis esset in ea quasi quaedam forma permanens, nisi haberet intellectum; ita rei naturali potuit conferri virtus propria, ut forma in ipsa permanens, non autem vis qua agit ad esse ut instrumentum primae causae (...).

84 Thomas d'Aquin, Summa theologiae, I, 115.5, c.: (...) si sint multa agentia ordinate, semper secundum agens agit in virtute primi; nam primum agens movet secundum ad agendum. Et secundum hoc, omnia agunt in virtute ipsius Dei; et ita ipse est causa actionum omnium agentium. 
sujets qui effectuent l' action, l'agent particulier, cause seconde prochaine, est cause la plus conjointe à l'effet. Mais si nous considérons les pouvoirs requis par l' action, le pouvoir de la cause supérieure, présent dans l' agent particulier, est plus immédiatement cause de l'effet que le pouvoir de la cause inférieure, parce qu'il donne l' existence, sans laquelle, bien évidemment, l' effet ne serait rien (c' est-à-dire qu' il n'y aurait pas du tout d' effet de la cause seconde) ${ }^{85}$. En ce sens, la cause première est bien cause la plus profonde de l' effet ${ }^{86}$.

En conclusion, on voit qu'il n' est pas exact de dire, comme on le fait souvent, que selon Thomas les créatures ne peuvent pas donner l'existence ${ }^{87}$. Les créatures ne peuvent pas donner l'existence par leur vertu propre; et le pouvoir divin de créer ne peut non plus leur être délégué. Mais lorsqu' elles sont impliquées dans un processus de génération, la causalité divine se combine à leur propre causalité en une action unique qui donne l'existence. Il vaut la peine de relire sous cet éclairage certains passages que nous avons étudiés. L'existence est l'effet propre de la cause première, toutefois Thomas dit clairement qu' elle est aussi l' effet des autres agents, qui la causent également dans la mesure où ils agissent par le pouvoir de la cause première ${ }^{88}$.

85 Thomas d'Aquin, De potentia, 3.7, c.: Sic ergo si consideremus supposita agentia, quodlibet agens particulare est immediatum ad suum effectum. Si autem consideremus virtutem qua fit actio, sic virtus superioris causae erit immediatior effectui quam virtus inferioris. Cf. De veritate, 3.7, c.: Sed quia nos ponimus Deum immediatam causam uniuscuiusque rei secundum quod in omnibus causis secundis operatur (...). Noter toutefois la nuance introduite par un quasi dans Summa contra Gentiles, III, 70.5: Oportet ergo quod actio inferioris agentis non solum sit ab eo per virtutem propriam, sed per virtutem omnium superiorum agentium: agit enim in virtute omnium. Et sicut agens infimum invenitur immediatum activum, ita virtus primi agentis invenitur immediata ad producendum effectum: nam virtus infimi agentis non habet quod producat hunc effectum ex se, sed ex virtute proximi superioris; et virtus illius hoc habet ex virtute superioris; et sic virtus supremi agentis invenitur ex se productiva effectus, quasi causa immediata; sicut patet in principiis demonstrationum, quorum primum est immediatum.

86 Thomas d'Aquin, De veritate, 8.16, ad 12: (...) ipse Deus est propria et immediata causa uniuscuiusque rei, et quodammodo magis intima unicuique quam ipsum sit intimum sibi, ut Augustinus dicit. Thomas d'Aquin, Summa contra Gentiles, III, 67.5: Causa autem actionis magis est illud cuius virtute agitur quam etiam illud quod agit: sicut principale agens magis quam instrumentum. Deus igitur principalius est causa cuiuslibet actionis quam etiam secundae causae agentes.

87 Encore récemment, Baldner 2017, p. 157. En revanche, Te Velde 1995, p. 176-177, et Wippel 2000 avait déjà attiré l' attention sur ce point.

88 Thomas d'Aquin, Summa contra Gentiles, III, 66.6: (...) esse est proprius effectus primi agentis, et omnia alia agunt ipsum inquantum agunt in virtute primi agentis (je souligne). Thomas d'Aquin, De potentia, 7.2, c.: Omnes autem causae creatae communicant in uno effectu qui est esse, licet singulae proprios effectus habeant, in quibus distinguuntur. Calor enim facit calidum esse, et aedificator facit domum esse. Conveniunt ergo in hoc quod cau- 
Aucune créature, dit-il également, ne donne l'existence si ce n' est en participant de l'action de Dieu; mais cela signifie donc que les créatures donnent bien l' existence, même si ce n' est qu' en participant de l' action de Dieu - c'est pour cela, explique Thomas, que le De causis affirme que l'Intelligence et l' Âme ont une opération divine en tant qu' elles donnent l'existence ${ }^{89}$. Les agents créés ne produisent pas l'être absolument parlant, mais expliquent que l' existence soit reçue en telle ou telle chose ${ }^{90}$. C'est qu' il n'y a pas deux axes de causalité, l'un par lequel une substance actualise une forme spécifique dans la potentialité de la matière, et l' autre par lequel Dieu serait seule cause efficiente de l' existence du composé forme / matière ${ }^{91}$. Cela serait nier la causalité efficiente des créatures. Les causes créées, chez Thomas, sont de réels agents, qui font leur part de travail dans la production d'autres créatures. Cependant, dans l'étiologie proclusienne transmise par le De causis comme dans le modèle tiré de la causalité instrumentale par Thomas, l'activité d'une cause supérieure peut s' exercer, non pas en parallèle, mais dans et par celle de causes secondes. Une seule forme est actualisée dans l' effet, par le dernier agent (c' est-à-dire la cause instrumentale prochaine, par exemple le géniteur dans un processus de reproduction), mais cette forme contient tout ce que les agents supérieurs concourant ont à contribuer, dont l'existence (forma dat esse) ${ }^{92}$. Ces derniers agissent non pas seulement sur l' effet de la cause instrumentale pour le rendre plus parfait, mais sur la cause instrumentale elle-même pour la faire agir plus parfaitement ${ }^{93}$. L'action d'une créature, via la forme qu' elle actualise,

sant esse, sed differunt in hoc quod ignis causat ignem, et aedificator causat domum (je souligne).

89 Thomas d'Aquin, De potentia, 3.1, c.: (...) nulla res dat esse, nisi in quantum est in ea participatio divinae virtutis. Propter quod etiam dicitur in Libro de causis, quod anima nobilis habet operationem divinam in quantum dat esse (je souligne). Thomas d'Aquin, De potentia, 3.7, c.: (...) ut dicitur in Libro de causis, intelligentia non dat esse, nisi prout est in ea virtus divina. Thomas d'Aquin, Summa theologiae, I, 45.5, c.: Unde etiam dicitur Liber de causis quo neque intelligentia vel anima nobilis dat esse, nisi inquantum operatur operatione divina.

90 Thomas d'Aquin, Summa theologiae, I, 45.5, ad 1: Nullum igitur ens creatum potest producere aliquod ens absolute, nisi inquantum esse causat in hoc (...).

91 Contrairement à ce que dit Baldner 2017, p. 155-156. Voir Litt 1963, p. 176-177.

92 Thomas d'Aquin, Quaestio disputata de spiritualibus creaturis 3 , ad 20 : (...) ultimum agens proprium agit in virtute omnium superiorum. Unde non imprimuntur a diversis agentibus diversae formae in uno individuo, sed una forma est quae imprimitur a proximo agente, continens in se virtute omnes formas praecedentes.

93 Contrairement à ce qu'écrit Dvořák 2013, p. 626: «There is no implication that the secondary agent has to be causally affected by the primary cause in order to do its causal job, i.e. produce its effect. The primary cause affects the patient only and is responsible solely for the actuality of the form in the patient». Au-delà de la question de la nature exacte de la comparaison des causes instrumentales naturelles avec des outils (artéfacts), cela va 
peut donc porter l' esse jusqu' à l' effet, avec les autres propriétés, particulières, qu' elle lui donne. La concurrence des causes supérieures, y compris la donation

tout simplement à l' encontre de ce que Thomas explique dans De potentia, 3.7, c. Premièrement, il dit que l'influence divine doit assister l' agent créé tout comme l'influence des corps célestes assiste les éléments terrestres dans leurs actions (Sic ergo oportet virtutem divinam adesse cuilibet rei agenti, sicut virtutem corporis caelestis oportet adesse cuilibet corpori elementari agenti). Or, les corps célestes agissent non seulement sur les corps qui reçoivent l' action des éléments, mais sur les éléments eux-mêmes, comme on le voit dans les processus de génération, où le pouvoir des corps célestes est dit résider dans les pouvoirs élémentaux, pour les doper, pour ainsi dire, et leur faire faire ce qu' ils ne pourraient faire par eux-mêmes, à savoir engendrer une chose vivante (cf. Scriptum super Sententiis, II, 18.2.3, ad 2: [...] ex actionibus qualitatum activarum consequitur anima sensibilis et aliae formae substantiales secundum quod in eis ut instrumentis manet virtus animae, vel alterius substantialis formae et ipsius caeli; et ideo non oportet, cum non agant in virtute sua tantum, quod ex actionibus earum nihil sequatur ultra earum speciem: quia ex motu instrumenti sequitur effectus secundum rationem principalis agentis [je souligne]. Voir aussi Thomas d'Aquin, Summa theologiae, I, 71.1, ad 1: Non quod aqua aut terra habeat in se virtutem producendi omnia animalia, ut Avicenna posuit, sed quia hoc ipsum quod ex materia elementari, virtute seminis vel stellarum, possunt animalia produci, est ex virtute primitus elementis data). Il en va de même pour leur action sur les corps mixtes: ils agissent soit simplement par mouvement, comme la lune cause les marées - ce qui n' est pas pertinent ici -, soit en imprimant une forme dans ces corps mixtes pour leur donner un pouvoir d' agir qu' ils n' ont pas par eux-mêmes (De operationibus occultis naturae: [...] huiusmodi actiones consequantur aliquas formas uel uirtutes impressas corporibus elementatis a superioribus agentibus). Le cas de l' aimant est un exemple que Thomas cite souvent; or, ce n' est pas sur le fer que l'influence des cieux agit, mais sur l' aimant pour qu'il attire le fer (De veritate 5.10, ad 5, 22.13.c. Summa contra Gentiles, II, 68 et III, 92). Thomas dit encore en De potentia 3.7, c. qu' un individu qui procrée un autre individu est la cause instrumentale des corps célestes en ce qui concerne la nature spécifique transmise (Hoc ergo individuum agendo non potest constituere aliud in simili specie nisi prout est instrumentum illius causae, quae respicit totam speciem et ulterius totum esse naturae inferioris. Et propter hoc nihil agit ad speciem in istis inferioribus nisi per virtutem corporis caelestis [...]. Cf. Summa theologiae, 115.3, ad 2, et Quaestio disputata de malo 16.9, c.: Nam corpora caelestia sunt principia generationis hominum, et aliorum animalium perfectorum, mediante virtute particulari, quae est in seminibus [...] [je souligne]). Or, il serait absurde de dire que l' individu ne transmet pas cette nature spécifique, comme si un chien n' engendrait pas un chien mais, bien plutôt, les corps célestes donnaient directement la nature canine à sa progéniture et le chien ne lui donnait que des caractéristiques individuelles. En fait, l' action des corps célestes s' exercent à travers le chien, qui à la fois transmet la nature canine (qui ne vient pas de lui) et des caractéristiques individuelles (qui viennent de lui). Voir Summa theologiae I, 118.1, ad 3: dans l' espèce de ferment (spiritus) qui se trouve dans le semen est quidam calor ex virtute caelestium corporum, quorum etiam virtute agentia inferiora agunt ad speciem, ut supra dictum est. Et quia in huiusmodi spiritu concurrit virtus animae cum virtute caelesti, dicitur quod homo generat hominem, et sol (cf. Aristote, Physica, II, 2, 194b13). Ici encore, le pouvoir d'un corps céleste agit non pas directement sur l' effet de la cause instrumentale, mais dans la cause instrumentale même (voir aussi Scriptum super Sententiis II, 18.2.3, ad 5 : 
d' existence par Dieu, s' exerce à travers la forme que donne l' agent particulier à son effet prochain.

Certes, Thomas écrit souvent que Dieu seul peut créer. Mais cela s'entend de la donation d'existence ex nihilo. C'est pour cela que Thomas rejette l' idée, non seulement d'Avicenne, mais aussi de Pierre Lombard ${ }^{94}$, ou du Liber de causis lui-même, que bien que créer soit l'action propre de la cause première, néanmoins les causes secondes peuvent créer dans la mesure où elles agissent par le pouvoir de la cause première. La position de Pierre Lombard, pourtant, s'appuie précisément sur le principe que nous venons de voir: il est possible que quelque chose participe instrumentalement à l' action causale propre d'une autre chose, c' est-à-dire cause non pas en vertu de son pouvoir mais en vertu du pouvoir de cette autre chose ${ }^{95}$. Contradiction de Thomas, donc? Non, car il est question de création, c' est-à-dire de donner l' existence ex nihilo. Comme le précise Thomas, une cause instrumentale ne participe à l'action d'une cause supérieure que dans la mesure où, par son pouvoir propre, elle

〈in animalia perfectiora > oportet quod cum virtute caelesti adsit in semine virtus animae a patre derivata: est enim virtus caelestis in omnibus corporibus inferioribus sicut virtus motoris in moto [...] [je souligne]. Et Super Librum de causis expositio, p. 38, 1. 27-30: [...] ipsum corpus humanum disponitur ad hoc quod sit susceptivum talis animae pervirtutem caelestis corporis operantem in semine, ratione cuius dicitur quod homo generat hominem et sol [je souligne]). Enfin, les corps célestes sont à leur tour instruments des substances intellectuelles pour la transmission des formes substantielles aux choses terrestres, et Thomas dit de même clairement que leur influence réside dans les corps célestes agents comme c' est le cas pour tout instrument (Scriptum super Sententiis, II, d. 15, q. 1, a. 2, c.: Relinquitur autem virtus spiritualis substantiae in motu corporis caelestis, ad modum quo virtus motoris relinquitur in instrumento: et per hunc modum omnes formae naturales descendunt a formis quae sunt sine materia [...] sicut formae artificiatorum ab artifice [je souligne]. De potentia, 3.11, ad 14: [...] virtus substantiae virtualis moventis relinquitur in corpore caelesti et motu eius, non sicut forma habens esse completum in natura, sed per modum intentionis, sicut virtus artis est in instrumento artificis). Pareillement, donc, on doit dire que Dieu agit dans tout agent créé ([...] potest dici quod Deus in qualibet re operatur in quantum eius virtute quaelibet res indiget ad agendum [...] [je souligne]), et que toute créature agit comme cause instrumentale par le pouvoir de Dieu (De potentia, 3.7, c.: Sic ergo Deus est causa omnis actionis, prout quodlibet agens est instrumentum divinae virtutis operantis [...] Sic ergo Deus est causa actionis cuiuslibet in quantum dat virtutem agendi, et in quantum conservat eam, et in quantum applicat actioni, et in quantum eius virtute omnis alia virtus agit [je souligne]).

Thomas d'Aquin, Scriptum super Sententiis, Iv, d. 5, q. 3, a. 3 .

95 Thomas d'Aquin, Summa theologiae, I, 45.5, c.: Contingit autem quod aliquid participet actionem propriam alicuius alterius, non virtute propria, sed instrumentaliter, inquantum agit in virtute alterius; sicut aer per virtutem ignis habet calefacere et ignire. Et secundum hoc, aliqui opinati sunt quod, licet creatio sit propria actio universalis causae, tamen aliqua inferiorum causarum inquantum agit in virtute primae causae, potest creare. 
prépare et dispose le sujet récepteur à recevoir l' effet propre de la cause supérieure ${ }^{96}$. Toutes les causes instrumentales font ce travail de préparation ${ }^{97}$. Certaines vont plus loin et contribuent directement à l' effet de la cause principale, comme nous l'avons vu; mais même ces dernières ont une action préparatrice sur le sujet de l' action de la cause principale. C' est en coupant le bois (ce qui est l'action préparatoire) qu' une scie introduit la forme de banc (ce qui est l' action finale $)^{98}$. Or, dans une situation où il n'y aurait rien sinon la cause première et cette cause seconde, il n'y aurait pas de sujet sur lequel cette cause seconde pourrait exercer sa propre action causale à titre de préparation. C' est pourquoi les créatures ne peuvent pas créer, c' est-à-dire produire ex nihilo, même si Dieu leur prêtait sa puissance ${ }^{99}$. En revanche, lorsqu'une matière est donnée (comme c'est le cas dans le cours ordinaire de la Nature), rien n' empêche qu' une créature ne joue le rôle de cause instrumentale à l'égard de la donation d'existence ${ }^{100}$. Par son action propre, elle prépare et informe un sujet récepteur, qui est actualisé en un composé rendu existant par l'action propre de Dieu, laquelle se communique par l'intermédiaire de cette même cause seconde.

On voit donc que Thomas n'hésite pas à corriger le Livre des Causes quand il le doit ${ }^{101}$. La thèse selon laquelle la cause première crée «l'âme, la nature et les autres choses par l' entremise de l' intelligence» (VIII, 87) est jugée fausse ou correcte selon le contexte. Fausse si l'on parle de création au sens strict,

96 Thomas d'Aquin, Summa theologiae, I, 45.5, c.: (...) causa secunda instrumentalis non participat actionem causae superioris, nisi inquantum per aliquid sibi proprium dispositive operatur ad effectum principalis agentis. (...) Unde non potest aliquid operari dispositive et instrumentaliter ad hunc effectum, cum creatio non sit ex aliquo praesupposito, quod possit disponi per actionem instrumentalis agentis. Sic igitur impossibile est quod alicui creaturae conveniat creare, neque virtute propria, neque instrumentaliter sive per ministerium.

97 Voir Solère 2019, p. 163-167.

98 Thomas d'Aquin, Summa theologiae, I, 45.5, c.: (...) securis, scindendo lignum, quod habet ex proprietate suae formae, producit scamni formam, quae est effectus proprius principalis agentis (je souligne). Voir aussi supra, n. 42.

99 Voir aussi Thomas d'Aquin, De potentia, 4.3, c.: Nam actio alicuius, etiamsi sit eius ut instrumenti, oportet ut ab eius potentia egrediatur. Cum autem omnis creaturae potentia sit finita, impossibile est quod aliqua creatura ad creationem operetur, etiam quasi instrumentum. Nam creatio infinitam virtutem requirit in potentia a qua egreditur.

100 Thomas d'Aquin, De potentia, 3.8, c.: Nihil ergo obstat per hoc quod dicitur quod per naturam ex nihilo nihil fit, quin formas substantiales ex operatione naturae esse dicamus. Nam id quod fit, non est forma, sed compositum; quod ex materia fit, et non ex nihilo. Et fit quidem ex materia, in quantum materia est in potentia ad ipsum compositum, per hoc quod est in potentia ad formam. Pour le cas particulier de l'âme humaine, voir Summa contra Gentiles, II, 89.16: Sic enim homo sibi simile in specie generat, inquantum virtus seminis eius dispositive operatur ad ultimam formam, ex qua homo speciem sortitur.

Voir aussi Te Velde 1995, p. 171-175. 
c' est-à-dire ex nihilo ${ }^{102}$. Dans ce contexte, Thomas la juge même sévèrement, indiquant qu' elle est une source d'idolâtrie ${ }^{103}$. Mais si l' on parle des processus naturels, dans lesquels les causes secondes agissent sur un substrat préexistant, alors Thomas la reprend à son compte, et soutient qu' elles participent à l' action divine qui donne l' existence.

\section{Bibliographie}

\section{Sources primaires}

Liber de causis. Édition et traduction par O. Boulnois, P. Magnard, B. Pinchard, et J.L. Solère, Paris, Vrin, 1990.

Proclus, The Elements of Theology, éd., trad., introd. et commentaire par E.R. Dodds, Oxford, Clarendon Press, $1963^{2}$.

Franciscus Suárez, Disputationes metaphysicae, in Opera omnia, t. 25-26, ed. C. Berton, Paris, Louis Vivès, 1861.

Thomas d'Aquin, Opera omnia: www.corpusthomisticum.org/iopera.html.

Thomas d'Aquin, Super Librum de causis expositio, ed. H.-D. Saffrey, Fribourg, Société Philosophique / Louvain, E. Nauwelaerts, 1954.

\section{Littérature secondaire}

Baldner, S. (2017), «Thomas Aquinas and Francisco Suárez on the Problem of Concurrence », in Proceedings of the American Catholic Philosophical Association 9o, p. 149161.

D’Ancona, C. (2014), «The Liber de causis», in S. Gersh (éd.), Interpreting Proclus from Antiquity to the Renaissance, Cambridge, Cambridge University Press, p. 137-162.

Dvořák, P. (2013), «The Concurrentism of Thomas Aquinas: Divine Causation and Human Freedom», dans Philosophia 41, p. 617-634.

Freddoso, A. (1988), «Medieval Aristotelianism and the Case against Secondary Causation in Nature», dans T.V. Morris (eds), Divine and Human Action: Essays in the Metaphysics of Theism, Cornell, Cornell University Press, p. 74-118.

102 Thomas d'Aquin, De potentia, 3.4, ad 10: (...) error iste expresse in libro de causis invenitur, quod creaturae inferiores creatae sunt a Deo superioribus mediantibus: unde in hoc auctoritas illius non est recipienda.

103 Thomas d'Aquin, De potentia, 3.4, c.: Et per hunc modum posuerunt quidam philosophi, quod intelligentiae primae sunt creatrices secundarum, in quantum dant eis esse per virtutem causae primae in eis existentem. Nam esse per creationem, bonum vero et vita et huiusmodi, per informationem, ut in Libro de causis habetur. Et hoc fuit idolatriae principium, dum ipsis creatis substantiis quasi creatricibus aliarum, latriae cultus exhibebatur. 
Freddoso, A. (1991), «God's General Concurrence with Secondary Causes: Why Conservation is not Enough», dans Philosophical Perspectives 5, p. 553-585.

Freddodo, A. (1994), «God's General Concurrence with Secondary Causes: Pitfalls and Prospects », in American Catholic Philosophical Quarterly 67 (2), p. 131-156.

Litt, Th. (1963), Les Corps célestes dans l'univers de Saint Thomas d'Aquin, Louvain / Paris, Publications Universitaires / Béatrice-Nauwelaerts.

Mancha, L.A.Jr. (2012), «Aquinas, Suárez, and Malebranche on Instrumental Causation and Premotion», dans International Philosophical Quarterly, 52 (3), p. 335-353.

Régnon, Th. de (19o6), La métaphysique des causes d'après Saint Thomas et Albert le Grand, 2 éd., Paris, Victor Retaux.

Solère, J.-L. (2019), «Duns Scotus versus Thomas Aquinas on Instrumental Causality», dans Oxford Studies in Medieval Philosophy 7, p. 147-185.

Te Velde, R. (1995), Participation and Substantiality in Thomas Aquinas, Leiden, Brill.

Torrell, J.-P. (1996), Saint Thomas Aquinas, vol. 1, trad. Robert Royal, Washington, D.C., The Catholic University of America Press.

Wippel, J. (2000), «Thomas Aquinas on Creatures as Causes of Esse», dans International Philosophical Quarterly Vol. XL-2, Issue 158, p. 197-213. 